\title{
NONPARAMETRIC TRANSFORMATION REGRESSION WITH NONSTATIONARY DATA
}

\author{
OLIVER LINTON \\ University of Cambridge \\ QIYING WANG \\ University of Sydney
}

\begin{abstract}
We examine a kernel regression estimator for time series that takes account of the error correlation structure as proposed by Xiao et al. (2003, Journal of the American Statistical Association 98, 980-992). We show that this method continues to improve estimation in the case where the regressor is a unit root or a near unit root process.
\end{abstract}

\section{INTRODUCTION}

This paper is concerned with estimation of a nonstationary nonparametric cointegrating regression. The theory of linear cointegration is extensive and originates with the work of Engle and Granger (1987) (see also Stock, 1987; Johansen, 1988; Phillips, 1991). Wang and Phillips (2009a, 2009b, 2011) recently considered the nonparametric cointegrating regression. They analyze the behavior of the standard kernel estimator of the cointegrating relation/nonparametric regression when the covariate is nonstationary. They showed that under self (random) normalization, the estimator is asymptotically normal. See also Phillips and Park (1998), Karlsen and Tjøstheim (2001), Karlsen, Myklebust, and Tjøstheim (2007), Schienle (2008), and Cai, Li, and Park (2009).

We extend this work by investigating an improved estimator in the case where there is autocorrelation in the error term. Standard kernel regression smoothers do not take account of the correlation structure in the covariate $x_{t}$ or the error process $u_{t}$ and estimate the regression function in the same way as if these processes were independent. Furthermore, the variance of such estimators is proportional to the short-run variance of $u_{t}, \sigma_{u}^{2}=\operatorname{var}\left(u_{t}\right)$, and does not depend on the regressor or error autocovariance functions $\gamma_{x}(j)=\operatorname{cov}\left(x_{t}, x_{t-j}\right), \gamma_{u}(j)=\operatorname{cov}\left(u_{t}, u_{t-j}\right), j \neq 0$. Although the time series properties do not affect the asymptotic variance of the usual nonparametric estimators, the error structure can be used to construct a more

\footnotetext{
The authors thank Professor Phillips and three referees for their very helpful comments on the original version. Linton acknowledges the European Research Council for financial support and Wang acknowledges research support from the Australian Research Council. Address correspondence to Oliver Linton, Faculty of Economics, University of Cambridge, Austin Robinson Building, Sidgwick Avenue, Cambridge CB3 9DD, United Kingdom; e-mail: obl20@cam.ac.uk.
} 
efficient estimator. Xiao, Linton, Carroll, and Mammen (2003) proposed a more efficient estimator of the regression function based on a prewhitening transformation. The transform implicitly takes account of the autocorrelation structure. They obtained an improvement in terms of variance over the usual kernel smoothers. Linton and Mammen (2008) proposed a type of iterated version of this procedure and showed that it attains higher efficiency. Both these contributions assumed that the covariate process was stationary and weakly dependent. We consider here the case where $x_{t}$ is nonstationary: of the unit root or close to unit root type. We allow the error process to have some short term memory, which is certainly commonplace in the linear cointegration literature. We show that the Xiao et al. (2003) procedure can improve efficiency even in this case and one still obtains asymptotic normality for the self-normalized estimator, which allows standard inference methods to be applied. In order to establish our results we require a new strong approximation result and use this to establish the $L_{2}$ convergence rate of the usual kernel estimator.

\section{THE MODEL AND MAIN RESULTS}

Consider a nonlinear cointegrating regression model

$y_{t}=m\left(x_{t}\right)+u_{t}, \quad t=1,2, \ldots, n$,

where $u_{t}=\rho u_{t-1}+\epsilon_{t}$ with $|\rho|<1$ and $x_{t}$ is a nonstationary regressor. The conventional kernel estimator of $m(x)$ is defined as

$\hat{m}(x)=\frac{\sum_{s=1}^{n} y_{s} K\left[\left(x_{s}-x\right) / h\right]}{\sum_{s=1}^{n} K\left[\left(x_{s}-x\right) / h\right]}$,

where $K(\cdot)$ is a nonnegative real function and the bandwidth parameter $h \equiv h_{n} \rightarrow 0$ as $n \rightarrow \infty$. Applying the Cochrane-Orcutt transformation to (2.1), we obtain

$y_{t}-\rho y_{t-1}+\rho m\left(x_{t-1}\right)=m\left(x_{t}\right)+\epsilon_{t}$.

It is expected that a two-step estimator of $m(x)$ by using (2.2) may achieve efficiency improvements over the usual estimator $\hat{m}(x)$ that uses (2.1). The strategy to provide the two-step estimator is as follows:

Step 1: Construct an estimator of $\rho$ by

$\widehat{\rho}=\frac{\sum_{s=2}^{n} \widehat{u}_{s} \widehat{u}_{s-1}}{\sum_{s=2}^{n} \widehat{u}_{s-1}^{2}}$,

where $\widehat{u}_{t}=y_{t}-\hat{m}\left(x_{t}\right)$.

Step 2: Construct an estimator of $m(x)$, say $\hat{m}_{1}(x)$, by using (2.2) and the kernel method, but replace the unknown quantity $m(x)$ in (2.2) by $\hat{m}(x)$.

We now have a two-step estimator $\hat{m}_{1}(x)$ of $m(x)$, defined as follows:

$\hat{m}_{1}(x)=\frac{\sum_{t=1}^{n}\left[y_{t}-\widehat{\rho} y_{t-1}+\widehat{\rho} \hat{m}\left(x_{t-1}\right)\right] K\left[\left(x_{t}-x\right) / h\right]}{\sum_{t=1}^{n} K\left[\left(x_{t}-x\right) / h\right]}$. 
To establish our claim that $\hat{m}_{1}(x)$ achieves efficiency improvements over the usual estimator $\hat{m}(x)$, we make the following assumptions.

Assumption 2.1. $x_{t}=\lambda x_{t-1}+\xi_{t},\left(x_{0} \equiv 0\right)$, where $\lambda=1+\tau / n$ with $\tau \leq 0$ being a constant and $\left\{\xi_{j}, j \geq 1\right\}$ is a linear process defined by

$\xi_{j}=\sum_{k=0}^{\infty} \phi_{k} v_{j-k}$

where $\phi_{0} \neq 0, \phi \equiv \sum_{k=0}^{\infty} \phi_{k} \neq 0$, and $\sum_{k=0}^{\infty}\left|\phi_{k}\right|<\infty$, and $\left\{v_{j},-\infty<j<\infty\right\}$ is a sequence of i.i.d. (independent and identically distributed) random variables with $E v_{0}=0, E v_{0}^{2}=1, E\left|v_{0}\right|^{2+\delta}<\infty$ for some $\delta>0$; the characteristic function $\varphi(t)$ of $v_{0}$ satisfies $\int_{-\infty}^{\infty}(1+|t|)|\varphi(t)| d t<\infty$.

Assumption 2.2. $u_{t}=\rho u_{t-1}+\epsilon_{t}$ with $|\rho|<1$ and $\epsilon_{0}=u_{0}=0$, where $\mathcal{F}_{n, t}=$ $\sigma\left(\epsilon_{0}, \epsilon_{1}, \ldots, \epsilon_{t}, x_{1}, \ldots, x_{n}\right)$ and $\left\{\epsilon_{t}, \mathcal{F}_{n, t}\right\}_{t=1}^{n}$ forms a martingale difference sequence satisfying, as $n \rightarrow \infty$ first, and then $m \rightarrow \infty$,

$\max _{m \leq t \leq n}\left|E\left(\epsilon_{t}^{2} \mid \mathcal{F}_{n, t-1}\right)-\sigma^{2}\right| \rightarrow 0, \quad$ a.s.,

where $\sigma^{2}$ is a given constant, and $\sup _{1 \leq t \leq n, n \geq 1} E\left(\left|\epsilon_{t}\right|^{q} \mid \mathcal{F}_{n, t-1}\right)<\infty$ a.s. (almost surely) for some $q>2$.

Assumption 2.3. (a) $\int_{-\infty}^{\infty} K(s) d s=1$ and $K(\cdot)$ has a compact support; (b) For any $x, y \in \mathbb{R},|K(x)-K(y)| \leq C|x-y|$, where $C$ is a positive constant; (c) For $p \geq 2$,

$\int y^{p} K(y) d y \neq 0, \quad \int y^{i} K(y) d y=0, \quad i=1,2, \ldots, p-1$.

Assumption 2.4. (a) There exist a $0<\beta \leq 1$ and $\alpha \geq 0$ such that

$|m(x+y)-m(x)| \leq C\left(1+|x|^{\alpha}\right)|y|^{\beta}$,

for any $x \in \mathbb{R}$ and $|y|$ sufficiently small, where $C$ is a positive constant; (b) For given fixed $x, m(x)$ has continuous $p+1$ derivatives in a small neighborhood of $x$, where $p \geq 2$ is defined as in Assumption 2.3(c).

We have the following main results.

THEOREM 2.1. Suppose that Assumptions 2.1, 2.2, 2.3(a), and 2.4(a) hold. Then, we have

$\left(\sum_{t=1}^{n} K\left[\left(x_{t}-x\right) / h\right]\right)^{1 / 2}[\hat{m}(x)-m(x)] \rightarrow_{D} N\left(0, \sigma_{1}^{2}\right)$, 
for any $h$ satisfying $n h^{2} \rightarrow \infty$ and $n h^{2+4 \beta} \rightarrow 0$, where $\sigma_{1}^{2}=\left(1-\rho^{2}\right)^{-1} \sigma^{2}$ $\int_{-\infty}^{\infty} K^{2}(s) d t$. If in addition Assumptions 2.3(c) and 2.4(b) hold, then

$$
\begin{aligned}
& \left(\sum_{t=1}^{n} K\left[\left(x_{t}-x\right) / h\right]\right)^{1 / 2}\left[\hat{m}(x)-m(x)-\frac{h^{p} m^{(p)}(x)}{p !} \int_{-\infty}^{\infty} y^{p} K(y) d y\right] \\
& \quad{ }_{D} N\left(0, \sigma_{1}^{2}\right),
\end{aligned}
$$

for any $h$ satisfying $n h^{2} \rightarrow \infty$ and $n h^{2+4 p}=O(1)$.

THEOREM 2.2. Suppose that Assumptions 2.1, 2.2, 2.3(a) and (b), 2.4(a), and $\sum_{i=0}^{\infty} i\left|\phi_{i}\right|<\infty$ hold. Then, we have

$\widehat{\rho}-\rho=O_{P}\left\{n^{\alpha / 2} h^{\beta}+\left(n h^{2}\right)^{-1 / 4}\right\}$,

and with $\sigma_{2}^{2}=\sigma^{2} \int_{-\infty}^{\infty} K^{2}(s) d t$,

$\left(\sum_{t=1}^{n} K\left[\left(x_{t}-x\right) / h\right]\right)^{1 / 2}\left[\hat{m}_{1}(x)-m(x)\right] \rightarrow_{D} N\left(0, \sigma_{2}^{2}\right)$,

for any $h$ satisfying that $n h^{2+4 \beta} \rightarrow 0, n^{\alpha} h^{2 \beta} \rightarrow 0$, and $n^{1-\epsilon_{0}} h^{2} \rightarrow \infty$ for some $\epsilon_{0}>0$. If in addition Assumptions 2.3(c) and 2.4(b) hold, then

$$
\begin{aligned}
& \left(\sum_{t=1}^{n} K\left[\left(x_{t}-x\right) / h\right]\right)^{1 / 2}\left[\hat{m}_{1}(x)-m(x)-\frac{h^{p} m^{(p)}(x)}{p !} \int_{-\infty}^{\infty} y^{p} K(y) d y\right] \\
& \quad \rightarrow_{D} N\left(0, \sigma_{2}^{2}\right),
\end{aligned}
$$

for any $h$ satisfying that $n h^{2+4 p}=O(1), n^{\alpha} h^{2 \beta} \rightarrow 0$, and $n^{1-\epsilon_{0}} h^{2} \rightarrow \infty$ for some $\epsilon_{0}>0$.

Remark 1. Theorem 2.1 generalizes certain related results in previous articles. See, for instance, Wang and Phillips (2009a, 2011), where the authors investigated the asymptotics under $\rho=0$ and $\tau=0$. As noted in previous works, the conditions on $m(x)$ to establish our results are quite weak, in particular, a wide range of regression functions $m(x)$ are included in Assumption 2.4(a), like $m(x)=1$ / $\left(1+\theta|x|^{\beta}\right), m(x)=\left(a+b e^{x}\right) /\left(1+e^{x}\right)$, and $m(x)=\theta_{1}+\theta_{2} x+\cdots+\theta_{k} x^{k-1}$.

If we are only interested in the asymptotics of $\hat{m}(x)$, recent research has shown that the information set $\mathcal{F}_{n, t}=\sigma\left(\epsilon_{0}, \epsilon_{1}, \ldots, \epsilon_{t}, x_{1}, \ldots, x_{n}\right)$ given in Assumption 2.2 can be reduced to $\mathcal{F}_{t}=\sigma\left(\epsilon_{0}, \epsilon_{1}, \ldots, \epsilon_{t}, x_{1}, \ldots, x_{t}\right)$, a more natural condition in the framework of cointegration. We refer to Wang and Phillips (2009a) and Wang (2014) for current developments. However, due to the involvement of $x_{1}, \ldots, x_{n}$ in the definition of $\hat{m}_{1}(x)$, it is difficult to weaken this restriction for the asymptotics of $\hat{m}_{1}(x)$, which is provided in Theorem 2.2. Theorem 2.1 of this paper is only stated to make a comparison with Theorem 2.2, which does not provide a general result in this direction. 
Remark 2. As $|\rho|<1$, Theorem 2.2 confirms the claim that $\hat{m}_{1}(x)$ achieves efficiency improvements over the usual estimator $\hat{m}(x)$ under certain additional conditions on $m(x)$ and the bandwidth $h$. Among these additional conditions, the requirement on the bandwidth $h$ (that is, $n^{\alpha} h^{2 \beta} \rightarrow 0$ and $n^{1-\epsilon_{0}} h^{2} \rightarrow \infty$, where $\epsilon_{0}$ can be sufficiently small) implies that $0 \leq \alpha<\beta$, which in turn requires that the rate at which $m(x)$ diverges to $\infty$ in the tail is not faster than $|x|^{1+\beta}$. In comparison with Theorem 2.1, this is a little bit restrictive but it is reasonable, due to the fact that the consistency result (2.6) heavily depends on the following uniform convergence

$\frac{1}{n} \sum_{t=1}^{n}\left[\hat{m}\left(x_{t}\right)-m\left(x_{t}\right)\right]^{2}=O_{P}\left(r_{n}\right)$

where $0<r_{n} \rightarrow 0$ is a sequence of constants. As $x_{t} \sim \sqrt{t}$ under our model, it is natural for the restriction on the tail of $m(x)$ to enable (2.9). The result (2.9) is a consequence of Theorem 3.1 in the next section, which provides a strong approximation result on the convergence to a local time process.

One referee argued that the condition $n^{\alpha} h^{2 \beta} \rightarrow 0$ can be weakened if the local linear estimator $\tilde{m}(x)$ is used in the definition of $\hat{m}_{1}(x)$ instead of $\hat{m}(x)$. Since this improvement requires new limit theorems, we leave the topic for future work.

Remark 3. Consider model (2.1) with $\operatorname{AR}(k)$ errors, i.e., $u_{t}$ is assumed to be strictly stationary satisfying

$u_{t}=\rho_{1} u_{t-1}+\rho_{2} u_{t-2}+\cdots+\rho_{k} u_{t-k}+\epsilon_{t}$,

where $\max _{1 \leq j \leq k}\left|\rho_{j}\right|<1$. In this situation, similar to (2.2), we have

$y_{t}-\sum_{j=1}^{k} \rho_{j}\left[y_{t-j}-m\left(x_{t-j}\right)\right]=m\left(x_{t}\right)+\epsilon_{t}$,

and, as in Step 2, we may construct a two-step estimator $\hat{m}_{2}(x)$ of $m(x)$ as

$\hat{m}_{2}(x)=\frac{\sum_{t=1}^{n}\left\{y_{t}-\sum_{j=1}^{k} \hat{\rho}_{j}\left[y_{t-j}-\hat{m}\left(x_{t-j}\right)\right]\right\} K\left[\left(x_{t}-x\right) / h\right]}{\sum_{t=1}^{n} K\left[\left(x_{t}-x\right) / h\right]}$,

where $\left(\hat{\rho}_{1}, \ldots, \hat{\rho}_{k}\right)^{\top}$ is a Least Squares (LS) estimator of $\hat{u}_{t}$ on $\hat{u}_{t-1}, \hat{u}_{t-2}, \ldots$, $\hat{u}_{t-k}$, where $\hat{u}_{t}=y_{t}-\hat{m}\left(x_{t}\right)$.

If $\epsilon_{j}, j \in Z$, are assumed to be i.i.d. random variables with $E \epsilon_{0}=0$ and $E \epsilon_{0}^{2}=\sigma^{2}$ and $\epsilon_{j}$ are independent of $x_{t},(2.4)$ and (2.5) hold true except that $\sigma_{1}^{2}$ is replaced by

$\sigma_{1}^{* 2}=\left(\sigma^{2}+\rho^{\top} \gamma\right) \int_{-\infty}^{\infty} K^{2}(s) d t$ 
where $\rho=\left(\rho_{1}, \ldots, \rho_{k}\right)^{\top}$ and $\gamma=\left(\gamma_{1}, \ldots, \gamma_{k}\right)^{\top}$ with $\gamma_{j}=\operatorname{cov}\left(u_{1}, u_{1+j}\right), 1 \leq j \leq k$. Furthermore, (2.7) and (2.8) hold for $\hat{m}_{1}(x)$ being replaced by $\hat{m}_{2}(x)$. Since we generally have $\rho^{\top} \gamma>0, \hat{m}_{2}(x)$ achieves efficiency improvements over $\hat{m}(x)$ under model (2.1) with $A R(k)$ errors (2.10). The proof of this claim is similar to Theorems 2.1 and 2.2, but involves some complicated calculations. The details are hence omitted.

\section{STRONG APPROXIMATION TO LOCAL TIME}

This section investigates the strong approximation to a local time process, which provides a technical tool in the development of the uniform convergence such as (2.9) for the kernel estimator $\hat{m}(x)$. As the conditions imposed are different, this section can be read separately.

Let $x_{k, n}, 1 \leq k \leq n, n \geq 1$ be a triangular array, constructed from some underlying nonstationary time series and assume that there is a continuous limiting Gaussian process $G(t), 0 \leq t \leq 1$, to which $x_{[n t], n}$ converges weakly, where $[a]$ denotes the integer part of $a$. In many applications, we let $x_{k, n}=d_{n}^{-1} x_{k}$, where $x_{k}$ is a nonstationary time series, such as a unit root or long memory process, and $d_{n}$ is an appropriate standardization factor. This section is concerned with the limiting behavior of the statistic $S_{n}(t)$, defined by

$$
S_{n}(t)=\frac{c_{n}}{n} \sum_{k=1}^{n} g\left[c_{n}\left(x_{k, n}-x_{[n t], n}\right)\right], \quad t \in[0,1],
$$

where $c_{n}$ is a certain sequence of positive constants and $g$ is a real integrable function on $\mathbb{R}$. As noticed in the last section and previous research (see, e.g., Wang and Phillips, 2012), this kind of statistic appears in the inference for the unknown regression function $m(x)$ and its limiting behavior plays a key role in related research fields.

The aim of this section is to provide a strong approximation result for the target statistic. To achieve our aim, we make use of the following assumptions.

Assumption 3.1. $\sup _{x}|x|^{\gamma}|g(x)|<\infty$ for some $\gamma>1, \int_{-\infty}^{\infty}|g(x)| d x<\infty$, and $|g(x)-g(y)| \leq C|x-y|$, whenever $|x-y|$ is sufficiently small on $\mathbb{R}$.

Assumption 3.2. On a rich probability space, there exists a continuous semimartingale $G(t)$ having a local time $L_{G}(t, s)^{1}$ such that, for some $\xi>0$,

$\left|L_{G}(1, s)-L_{G}(1, t)\right| \leq C|s-t|^{\xi} \quad$ a.s.

and a sequence of stochastic processes $G_{n}(t)$ such that $\left\{G_{n}(t) ; 0 \leq t \leq 1\right\}={ }_{D}$ $\{G(t) ; 0 \leq t \leq 1\}$ for each $n \geq 1$ and

$\sup \left|x_{[n t], n}-G_{n}(t)\right|=o_{a . s .}\left(n^{-\delta_{0}}\right)$

$0 \leq t \leq 1$

for some $0<\delta_{0}<1$. 
Assumption 3.3. For all $0 \leq j<k \leq n, n \geq 1$, there exist a sequence of $\sigma$-fields $\mathcal{F}_{k, n}$ (define $\mathcal{F}_{0, n}=\sigma\{\phi, \Omega\}$, the trivial $\sigma$-field) such that:

(i) $x_{j, n}$ are adapted to $\mathcal{F}_{j, n}$ and, conditional on $\mathcal{F}_{j, n},[n /(k-j)]^{d}\left(x_{k, n}-x_{j, n}\right)$, where $0<d<1$, has a density $h_{k, j, n}(x)$ satisfying that $h_{k, j, n}(x)$ is uniformly bounded by a constant $K$ and

(ii) $\sup _{u \in \mathbb{R}}\left|h_{k, j, n}(u+t)-h_{k, j, n}(u)\right| \leq C \min \{|t|, 1\}$, whenever $n$ and $k-j$ are sufficiently large and $t \in \mathbb{R}$.

Assumption 3.4. There is an $\epsilon_{0}>0$ such that $n^{-\epsilon_{0}} c_{n} \rightarrow \infty$ and $n^{-1+\epsilon_{0}} c_{n} \rightarrow 0$.

The following is our main result.

THEOREM 3.1. Suppose that Assumptions 3.1-3.4 hold. Then, on the same probability space as in Assumption 3.2, for any $l>0$, we have

$\sup _{0 \leq t \leq 1}\left|S_{n}(t)-\tau L_{n t}\right|=o_{P}\left(\log ^{-l} n\right)$,

where $\tau=\int_{-\infty}^{\infty} g(t) d t$ and $L_{n t}=\lim _{\epsilon \rightarrow 0} \frac{1}{2 \epsilon} \int_{0}^{1} I\left(\left|G_{n}(s)-G_{n}(t)\right| \leq \epsilon\right) d s$.

The rate obtained in (3.3) may not be optimal. We conjecture that the optimal rate should have the form $n^{-\delta_{1}}$, where $\delta_{1}>0$ is related to $\delta_{0}>0$ given in Assumption 3.2. We are not able to establish the optimal rate due to the technical difficulty. However, by noting $\left\{L_{n t} ; 0 \leq t \leq 1\right\}={ }_{D}\left\{L_{G}(1, G(t)) ; 0 \leq t \leq 1\right\}$ due to $\left\{G_{n}(t) ; 0 \leq t \leq 1\right\}={ }_{D}\{G(t) ; 0 \leq t \leq 1\}$, the result (3.3) is enough in many applications. To illustrate, we have the following theorem which provides the lower bound of $S_{n}(t)$ over $t \in[0,1]$. As a consequence, we establish the result (2.9) when $x_{t}$ satisfies Assumption 2.1.

THEOREM 3.2. Let $x_{t}$ be defined as in Assumption 2.1 with $\sum_{k=0}^{\infty} k\left|\phi_{k}\right|<\infty$. Let Assumptions 2.3(a) and (b) hold. Then, for any $\eta>0$, there exist $M_{1}>0$ and $n_{0}>0$ such that

$P\left(\inf _{s=1,2, \ldots, n} \sum_{t=1}^{n} K\left[\left(x_{t}-x_{s}\right) / h\right] \geq \sqrt{n} h / M_{1}\right) \geq 1-\eta$,

for all $n \geq n_{0}$ and $h$ satisfying that $h \rightarrow 0$ and $n^{1-\epsilon_{0}} h^{2} \rightarrow \infty$ for some $\epsilon_{0}>0$. Consequently, we have

$V_{n}:=\frac{1}{n} \sum_{t=1}^{n}\left[\hat{m}\left(x_{t}\right)-m\left(x_{t}\right)\right]^{2}=O_{P}\left\{n^{\alpha} h^{2 \beta}+\left(n h^{2}\right)^{-1 / 2}\right\}$,

that is, (2.9) holds true if in addition $n^{\alpha} h^{2 \beta} \rightarrow 0$. 


\section{EXTENSION}

We next propose another estimator that potentially can improve efficiency further, following the method of Linton and Mammen (2008). Note that we can write

$m(x)=\frac{1}{1+\rho^{2}} E\left[Z_{t}^{-}(\rho)-\rho Z_{t+1}^{+}(\rho) \mid x_{t}=x\right]$,

where: $Z_{t}^{-}(\rho)=y_{t}-\rho y_{t-1}+\rho m\left(x_{t-1}\right)$ and $Z_{t}^{+}(\rho)=y_{t}-\rho y_{t-1}-m\left(x_{t}\right)$. Let $\widehat{m}(),. \widehat{\rho}$ be initial consistent estimators of $m($.$) and \rho$, and let $\widehat{y}_{t}=\widehat{Z}_{t}^{-}(\widehat{\rho})-$ $\widehat{\rho} \widehat{Z}_{t+1}^{+}(\widehat{\rho})$, where: $\widehat{Z}_{t}^{-}(\rho)=y_{t}-\widehat{\rho} y_{t-1}+\widehat{\rho} \widehat{m}\left(x_{t-1}\right)$ and $\widehat{Z}_{t}^{+}(\rho)=y_{t}-\widehat{\rho} y_{t-1}-$ $\widehat{m}\left(x_{t}\right)$. Then let

$\widehat{m}_{e f f}(x)=\frac{1}{1+\widehat{\rho}^{2}} \frac{\sum_{s=1}^{n-1} \widehat{y}_{s} K\left[\left(x_{s}-x\right) / h\right]}{\sum_{s=1}^{n-1} K\left[\left(x_{s}-x\right) / h\right]}$.

We claim that the following result holds. The proof is similar to earlier results and is omitted.

THEOREM 4.1. Suppose in addition to Assumptions 2.1, 2.2, 2.3(a) and (b), and 2.4(a), that $\sum_{i=0}^{\infty} i\left|\phi_{i}\right|<\infty$ holds. Then, for any $h$ satisfying $n h^{2+4 \beta} \rightarrow 0$, $n^{\alpha} h^{2 \beta} \rightarrow 0$ and $n^{1-\epsilon_{0}} h^{2} \rightarrow \infty$ for some $\epsilon_{0}>0$, we have

$\left(\sum_{t=1}^{n} K\left[\left(x_{t}-x\right) / h\right]\right)^{1 / 2}\left[\hat{m}_{e f f}(x)-m(x)\right] \rightarrow_{D} N\left(0, \sigma_{3}^{2}\right)$,

where $\sigma_{3}^{2}=\left(1+\rho^{2}\right)^{-1} \int_{-\infty}^{\infty} K^{2}(s) d t$.

We have $\sigma_{3}^{2} \leq \sigma_{2}^{2} \leq \sigma_{1}^{2}$, and so $\widehat{m}_{e f f}(x)$ is more efficient (according to asymptotic variance) than $\widehat{m}_{2}(x)$, which itself is more efficient than $\widehat{m}(x)$.

\section{MONTE CARLO SIMULATION}

We investigate the performance of our procedure on simulated data. We chose a similar design to Wang and Phillips (2009b) except that we focus on error autocorrelation rather than contemporaneous endogeneity. We suppose that

$y_{t}=m\left(x_{t}\right)+\sigma u_{t}, u_{t}=\rho_{0} u_{t-1}+\varepsilon_{t}$

with $m(x)=x$ and $m(x)=\sin (x)$, where $x_{t}=x_{t-1}+\eta_{t}$, with $\eta_{t} \sim N(0,1)$, $\sigma=0.2$, and $\varepsilon_{t} \sim N(0,1)$; the two errors are mutually independent. We used the Epanechnikov kernel $K(u)=0.75\left(1-u^{2}\right) 1(|u| \leq 1)$ with the bandwidth $h=n^{-b c}$. We examine a range of values of $\rho_{0} \in\{-1,-0.9, \ldots, 0.9,0.95,1\}$ (although we only show the results for nonnegative values of $\rho_{0}$ ) and the bandwidth constant $b c \in(10 / 18,1 / 2,1 / 3,1 / 5,1 / 10\}$. We consider $n=500$, 
TABLE 1. $n=500$ and $m(x)=x$

\begin{tabular}{|c|c|c|c|c|c|c|c|c|c|c|c|c|}
\hline \multirow[b]{2}{*}{$\rho / b c$} & \multicolumn{6}{|c|}{$\widehat{m}_{2}$} & \multicolumn{6}{|c|}{$\widehat{m}_{1}$} \\
\hline & $10 / 18$ & $1 / 2$ & $1 / 3$ & $1 / 5$ & $1 / 6$ & $1 / 10$ & $10 / 18$ & $1 / 2$ & $1 / 3$ & $1 / 5$ & $1 / 6$ & $1 / 10$ \\
\hline 0.00 & 0.1613 & 0.1311 & 0.0621 & 0.0343 & 0.0302 & 0.0257 & 0.1613 & 0.1311 & 0.0621 & 0.0343 & 0.0302 & 0.0257 \\
\hline 0.10 & 0.1615 & 0.1313 & 0.0622 & 0.0344 & 0.0304 & 0.0260 & 0.1615 & 0.1313 & 0.0623 & 0.0345 & 0.0304 & 0.0260 \\
\hline 0.20 & 0.1619 & 0.1317 & 0.0626 & 0.0347 & 0.0307 & 0.0263 & 0.1620 & 0.1319 & 0.0628 & 0.0349 & 0.0308 & 0.0263 \\
\hline 0.30 & 0.1627 & 0.1325 & 0.0631 & 0.0351 & 0.0311 & 0.0268 & 0.1630 & 0.1329 & 0.0637 & 0.0355 & 0.0314 & 0.0269 \\
\hline 0.40 & 0.1639 & 0.1337 & 0.0640 & 0.0358 & 0.0317 & 0.0275 & 0.1645 & 0.1345 & 0.0650 & 0.0365 & 0.0323 & 0.0277 \\
\hline 0.50 & 0.1657 & 0.1355 & 0.0652 & 0.0367 & 0.0326 & 0.0284 & 0.1668 & 0.1370 & 0.0671 & 0.0380 & 0.0337 & 0.0289 \\
\hline 0.60 & 0.1686 & 0.1383 & 0.0672 & 0.0382 & 0.0341 & 0.0299 & 0.1705 & 0.1408 & 0.0704 & 0.0404 & 0.0359 & 0.0308 \\
\hline 0.70 & 0.1736 & 0.1432 & 0.0707 & 0.0409 & 0.0366 & 0.0324 & 0.1768 & 0.1475 & 0.0759 & 0.0445 & 0.0397 & 0.0342 \\
\hline 0.80 & 0.1836 & 0.1530 & 0.0778 & 0.0466 & 0.0422 & 0.0379 & 0.1894 & 0.1608 & 0.0872 & 0.0530 & 0.0477 & 0.0413 \\
\hline 0.90 & 0.2130 & 0.1823 & 0.1010 & 0.0661 & 0.0612 & 0.0564 & 0.2261 & 0.1998 & 0.1209 & 0.0796 & 0.0730 & 0.0644 \\
\hline 0.95 & 0.2717 & 0.2423 & 0.1538 & 0.1126 & 0.1068 & 0.1011 & 0.2969 & 0.2757 & 0.1909 & 0.1378 & 0.1288 & 0.1168 \\
\hline 1.00 & 3.1401 & 3.3402 & 3.3938 & 3.2839 & 3.2661 & 3.2429 & 3.3769 & 3.6514 & 3.7446 & 3.5232 & 3.4795 & 3.4196 \\
\hline
\end{tabular}


TABLE 2. $n=1,000$ and $m(x)=x$

\begin{tabular}{|c|c|c|c|c|c|c|c|c|c|c|c|c|}
\hline \multirow[b]{2}{*}{$\rho / b c$} & \multicolumn{6}{|c|}{$\widehat{m}_{2}$} & \multicolumn{6}{|c|}{$\widehat{m}_{1}$} \\
\hline & $10 / 18$ & $1 / 2$ & $1 / 3$ & $1 / 5$ & $1 / 6$ & $1 / 10$ & $10 / 18$ & $1 / 2$ & $1 / 3$ & $1 / 5$ & $1 / 6$ & $1 / 10$ \\
\hline 0.00 & 0.1610 & 0.1269 & 0.0522 & 0.0251 & 0.0213 & 0.0172 & 0.1610 & 0.1269 & 0.0522 & 0.0251 & 0.0213 & 0.0171 \\
\hline 0.10 & 0.1611 & 0.1270 & 0.0524 & 0.0253 & 0.0214 & 0.0174 & 0.1612 & 0.1271 & 0.0524 & 0.0253 & 0.0215 & 0.0174 \\
\hline 0.20 & 0.1616 & 0.1275 & 0.0527 & 0.0256 & 0.0217 & 0.0177 & 0.1617 & 0.1276 & 0.0529 & 0.0257 & 0.0218 & 0.0177 \\
\hline 0.30 & 0.1623 & 0.1282 & 0.0532 & 0.0259 & 0.0221 & 0.0181 & 0.1626 & 0.1286 & 0.0538 & 0.0263 & 0.0224 & 0.0182 \\
\hline 0.40 & 0.1635 & 0.1294 & 0.0540 & 0.0265 & 0.0226 & 0.0186 & 0.1642 & 0.1302 & 0.0551 & 0.0272 & 0.0232 & 0.0189 \\
\hline 0.50 & 0.1654 & 0.1311 & 0.0552 & 0.0273 & 0.0234 & 0.0194 & 0.1665 & 0.1327 & 0.0571 & 0.0286 & 0.0245 & 0.0199 \\
\hline 0.60 & 0.1683 & 0.1339 & 0.0570 & 0.0287 & 0.0247 & 0.0207 & 0.1703 & 0.1367 & 0.0603 & 0.0309 & 0.0266 & 0.0216 \\
\hline 0.70 & 0.1735 & 0.1388 & 0.0602 & 0.0310 & 0.0270 & 0.0229 & 0.1768 & 0.1435 & 0.0658 & 0.0347 & 0.0301 & 0.0246 \\
\hline 0.80 & 0.1838 & 0.1486 & 0.0668 & 0.0363 & 0.0320 & 0.0278 & 0.1899 & 0.1572 & 0.0769 & 0.0427 & 0.0375 & 0.0310 \\
\hline 0.90 & 0.2147 & 0.1784 & 0.0887 & 0.0543 & 0.0496 & 0.0449 & 0.2287 & 0.1979 & 0.1107 & 0.0682 & 0.0615 & 0.0525 \\
\hline 0.95 & 0.2762 & 0.2392 & 0.1386 & 0.0978 & 0.0922 & 0.0867 & 0.3041 & 0.2780 & 0.1814 & 0.1248 & 0.1153 & 0.1024 \\
\hline 1.00 & 6.1783 & 6.6740 & 6.9673 & 6.8136 & 6.7811 & 6.7500 & 6.6138 & 7.2842 & 7.5941 & 7.2184 & 7.1329 & 7.0197 \\
\hline
\end{tabular}


TABLE 3. $E \widehat{\rho}, m(x)=\sin (x)$

\begin{tabular}{|c|c|c|c|c|c|c|c|c|c|c|c|c|}
\hline \multirow[b]{2}{*}{$\rho / b c$} & \multicolumn{6}{|c|}{$n=1,000$} & \multicolumn{6}{|c|}{$n=500$} \\
\hline & $10 / 18$ & $1 / 2$ & $1 / 3$ & $1 / 5$ & $1 / 6$ & $1 / 10$ & $10 / 18$ & $1 / 2$ & $1 / 3$ & $1 / 5$ & $1 / 6$ & $1 / 10$ \\
\hline 0.00 & -0.0137 & -0.0138 & -0.0149 & -0.0163 & -0.0161 & -0.0124 & -0.0179 & -0.0181 & -0.0213 & -0.0230 & -0.0225 & -0.0179 \\
\hline 0.10 & 0.0310 & 0.0405 & 0.0637 & 0.0726 & 0.0741 & 0.0789 & 0.0270 & 0.0356 & 0.0549 & 0.0632 & 0.0649 & 0.0702 \\
\hline 0.20 & 0.0756 & 0.0948 & 0.1424 & 0.1616 & 0.1645 & 0.1702 & 0.0720 & 0.0893 & 0.1311 & 0.1494 & 0.1524 & 0.1585 \\
\hline 0.30 & 0.1204 & 0.1492 & 0.2212 & 0.2507 & 0.2550 & 0.2618 & 0.1169 & 0.1432 & 0.2075 & 0.2358 & 0.2401 & 0.2471 \\
\hline 0.40 & 0.1652 & 0.2037 & 0.3000 & 0.3400 & 0.3457 & 0.3538 & 0.1619 & 0.1971 & 0.2841 & 0.3225 & 0.3282 & 0.3363 \\
\hline 0.50 & 0.2100 & 0.2583 & 0.3791 & 0.4296 & 0.4368 & 0.4464 & 0.2070 & 0.2511 & 0.3608 & 0.4095 & 0.4167 & 0.4263 \\
\hline 0.60 & 0.2550 & 0.3131 & 0.4583 & 0.5196 & 0.5284 & 0.5398 & 0.2521 & 0.3052 & 0.4378 & 0.4971 & 0.5059 & 0.5173 \\
\hline 0.70 & 0.3003 & 0.3683 & 0.5380 & 0.6101 & 0.6206 & 0.6343 & 0.2974 & 0.3596 & 0.5152 & 0.5853 & 0.5959 & 0.6098 \\
\hline 0.80 & 0.3461 & 0.4241 & 0.6183 & 0.7015 & 0.7137 & 0.7303 & 0.3432 & 0.4144 & 0.5932 & 0.6745 & 0.6871 & 0.7043 \\
\hline 0.90 & 0.3930 & 0.4811 & 0.7003 & 0.7946 & 0.8088 & 0.8287 & 0.3902 & 0.4702 & 0.6730 & 0.7659 & 0.7807 & 0.8017 \\
\hline 0.95 & 0.4174 & 0.5109 & 0.7433 & 0.8430 & 0.8581 & 0.8799 & 0.4153 & 0.4996 & 0.7147 & 0.8135 & 0.8293 & 0.8523 \\
\hline 1.00 & 0.4466 & 0.5462 & 0.7903 & 0.8935 & 0.9093 & 0.9324 & 0.4399 & 0.5296 & 0.7567 & 0.8614 & 0.8781 & 0.9033 \\
\hline
\end{tabular}


TABLE 4. $\operatorname{stdc}(\widehat{\rho}), m(x)=\sin (x)$

\begin{tabular}{|c|c|c|c|c|c|c|c|c|c|c|c|c|}
\hline \multirow[b]{2}{*}{$\rho / b c$} & \multicolumn{6}{|c|}{$n=1,000$} & \multicolumn{6}{|c|}{$n=500$} \\
\hline & $10 / 18$ & $1 / 2$ & $1 / 3$ & $1 / 5$ & $1 / 6$ & $1 / 10$ & $10 / 18$ & $1 / 2$ & $1 / 3$ & $1 / 5$ & $1 / 6$ & $1 / 10$ \\
\hline 0.00 & 0.0334 & 0.0326 & 0.0327 & 0.0325 & 0.0325 & 0.0323 & 0.0474 & 0.0466 & 0.0467 & 0.0467 & 0.0467 & 0.0467 \\
\hline 0.10 & 0.0351 & 0.0345 & 0.0339 & 0.0333 & 0.0331 & 0.0328 & 0.0488 & 0.0481 & 0.0479 & 0.0475 & 0.0474 & 0.0472 \\
\hline 0.20 & 0.0381 & 0.0376 & 0.0356 & 0.0340 & 0.0337 & 0.0332 & 0.0510 & 0.0504 & 0.0493 & 0.0481 & 0.0479 & 0.0476 \\
\hline 0.30 & 0.0419 & 0.0417 & 0.0376 & 0.0346 & 0.0341 & 0.0335 & 0.0540 & 0.0534 & 0.0508 & 0.0486 & 0.0482 & 0.0477 \\
\hline 0.40 & 0.0466 & 0.0466 & 0.0398 & 0.0351 & 0.0344 & 0.0336 & 0.0577 & 0.0569 & 0.0525 & 0.0488 & 0.0482 & 0.0476 \\
\hline 0.50 & 0.0517 & 0.0519 & 0.0423 & 0.0354 & 0.0344 & 0.0334 & 0.0619 & 0.0609 & 0.0543 & 0.0488 & 0.0480 & 0.0472 \\
\hline 0.60 & 0.0573 & 0.0575 & 0.0448 & 0.0354 & 0.0341 & 0.0328 & 0.0666 & 0.0654 & 0.0561 & 0.0485 & 0.0474 & 0.0463 \\
\hline 0.70 & 0.0633 & 0.0635 & 0.0472 & 0.0350 & 0.0333 & 0.0316 & 0.0718 & 0.0701 & 0.0580 & 0.0479 & 0.0464 & 0.0449 \\
\hline 0.80 & 0.0697 & 0.0697 & 0.0498 & 0.0343 & 0.0322 & 0.0298 & 0.0777 & 0.0753 & 0.0599 & 0.0469 & 0.0449 & 0.0426 \\
\hline 0.90 & 0.0767 & 0.0765 & 0.0526 & 0.0337 & 0.0309 & 0.0274 & 0.0844 & 0.0820 & 0.0618 & 0.0454 & 0.0427 & 0.0395 \\
\hline 0.95 & 0.0803 & 0.0800 & 0.0544 & 0.0339 & 0.0307 & 0.0264 & 0.0877 & 0.0863 & 0.0628 & 0.0448 & 0.0417 & 0.0377 \\
\hline 1.00 & 0.0869 & 0.0860 & 0.0572 & 0.0347 & 0.0310 & 0.0253 & 0.0942 & 0.0929 & 0.0668 & 0.0461 & 0.0427 & 0.0377 \\
\hline
\end{tabular}




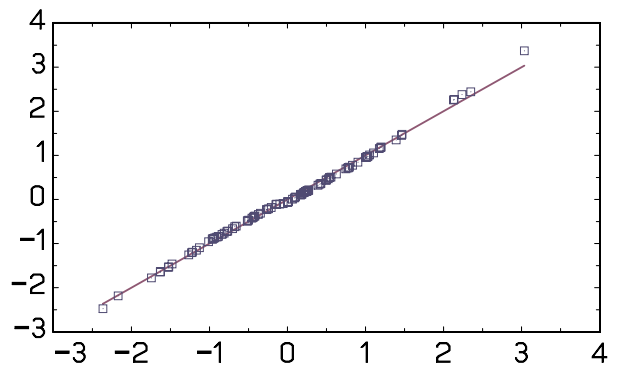

FIGURE 1. The QQ plot of the standardized estimator $\widehat{m}_{2}$.

1,000 and take $n s=1,000$ replications throughout. We report the performance measure

$A M S E=\frac{1}{K} \sum_{k=1}^{K}\left|\widehat{m}\left(x_{k}\right)-m\left(x_{k}\right)\right|^{2}$,

where $K=101$ and $x_{k}=\{-1,-0.98, \ldots, 1\}$. The results for the linear case are given in Tables 1 and 2. The results show that there is an improvement when going from $n=500$ to $n=1,000$ and when going from $\hat{m}$ to $\widehat{m}_{2}$. In the linear case, the bigger the bandwidth the better. In the cubic case (not shown), smaller bandwidths do better as the bias issue is much more severe in this case.

We show in Tables 3 and 4 the performance of the estimator of $\rho$ for $n=500$ and $n=1,000$. This varies with bandwidth and is generally quite poor, although improves with sample size. Finally, we give some indication of the distributional approximation. In Figure 1 we show the QQ plot for our (standardized) estimator $\widehat{m}_{2}$ in the case where $m(x)=\sin (x), \rho=0.95, n=1,000$, and $b c=1 / 10$.

\section{CONCLUSION}

We have shown that the main results of Xiao et al. (2003) regarding efficiency improvements can be extended to the case where the covariate process is nonstationary. In practice it may be important to take account of error autocorrelation when conducting inference about nonparametric cointegrating regressions. The idea of using the transformed model and the results obtained in terms of efficiency improvements are closely related to the augmented regression findings in Wang and Phillips (2009b), wherein it is shown that carrying the impact of endogeneity via the conditional mean in an augmented regression reduces the asymptotic variance of the kernel regression estimator.

\section{NOTE}

1. Here and below, we define $L_{G}(1, x)=\lim _{\epsilon \rightarrow 0} \frac{1}{2 \epsilon} \int_{0}^{1} I(|G(s)-x| \leq \epsilon) d s$, a local time process of the process $G(s)$, whenever it exists. 


\section{REFERENCES}

Cai, Z., Q. Li, \& J.Y. Park (2009) Functional-coefficient models for nonstationary time series data. Journal of Econometrics 148, 101-113.

Chan, N. \& Q. Wang (2014) Uniform convergence for Nadaraya-Watson estimators with nonstationary data. Econometric Theory 30, 1110-1133.

Chan, N.H. \& C.Z. Wei (1987) Asymptotic inference for nearly nonstationary AR(1) process. Annals of Statistics 15, 1050-1063.

Csörgö, M. \& P. Révész (1981) Strong Approximations in Probability and Statistics. Probability and Mathematical Statistics. Academic Press, Inc.

Engle, R.F. \& C.W.J. Granger (1987) Cointegration and error correction: Representation, estimation, and testing. Econometrica 55, 251-276.

Hall, P. \& C.C. Heyde (1980) Martingale Limit Theory and Its Application. Probability and Mathematical Statistics. Academic Press, Inc.

Johansen, S. (1988) Statistical analysis of cointegrating vectors. Journal of Economic Dynamics and Control 12, 231-254.

Karlsen, H.A. \& D. Tjøstheim (2001) Nonparametric estimation in null recurrent time series. Annals of Statistics 29, 372-416.

Karlsen, H.A., T. Myklebust, \& D. Tjøstheim (2007) Nonparametric estimation in a nonlinear cointegration model. Annals of Statistics 35, 252-299.

Linton, O.B. \& E. Mammen (2008) Nonparametric transformation to white noise. Journal of Econometrics 141, 241-264.

Liu, W., N. Chan, \& Q. Wang (2014) Uniform approximation to local time with applications in non-linear co-integrating regression. Preprint, School of Mathematics and Statistics, The University of Sydney. Available on http://www.maths.usyd.edu.au/u/pubs/publist/preprints/2014/liu-18.pdf.

Phillips, P.C.B. (1987) Towards a unified asymptotic theory for autoregression. Biometrika 74, 535-547.

Phillips, P.C.B. (1991) Optimal inference in cointegrated systems. Econometrica 59(2), 283-306.

Phillips, P.C.B. \& J.Y. Park (1998) Nonstationary Density Estimation and Kernel Autoregression. Cowles Foundation Discussion paper no. 1181.

Revuz, D. \& M. Yor (1994) Continuous Martingales and Brownian Motion. Fundamental Principles of Mathematical Sciences 293. Springer-Verlag.

Schienle, M. (2008) Nonparametric nonstationary regression. Unpublished Ph.D. thesis, University of Mannheim.

Stock, J.H. (1987) Asymptotic properties of least squares estimators of cointegration vectors. Econometrica 55, 1035-1056.

Wang, Q. (2014) Martingale limit theorem revisited and nonlinear cointegrating regression. Econometric Theory 30, 509-535.

Wang, Q. \& P.C.B. Phillips (2009a) Asymptotic theory for local time density estimation and nonparametric cointegrating regression. Econometric Theory 25, 710-738.

Wang, Q. \& P.C.B. Phillips (2009b) Structural nonparametric cointegrating regression. Econometrica 77, 1901-1948.

Wang, Q. \& P.C.B. Phillips (2011) Asymptotic theory for zero energy functionals with nonparametric regression applications. Econometric Theory 27, 235-259.

Wang, Q. \& P.C.B. Phillips (2012) A specification test for nonlinear nonstationary models. Annals of Statistics 40, 727-758.

Xiao, Z., O. Linton, R.J. Carroll, \& E. Mammen (2003) More efficient local polynomial estimation in nonparametric regression with autocorrelated errors. Journal of the American Statistical Association 98, 980-992. 


\section{APPENDIX: Proofs}

Appendix A.1 provides several preliminary lemmas. Some of them are of independent interest. The proofs of main theorems will be given in Appendixes A.2-A.4. Throughout the Appendix, we denote by $C_{0}, C, C_{1}, \ldots$ positive constants, which might be different at each appearance.

\section{A.1. Preliminary Lemmas}

First note that

$$
\begin{aligned}
x_{t} & =\sum_{j=1}^{t} \lambda^{t-j} \xi_{j}=\sum_{j=1}^{t} \lambda^{t-j} \sum_{i=-\infty}^{j} v_{i} \phi_{j-i} \\
& =\lambda^{t-s} x_{s}+\sum_{j=s+1}^{t} \lambda^{t-j} \sum_{i=-\infty}^{s} v_{i} \phi_{j-i}+\sum_{j=s+1}^{t} \lambda^{t-j} \sum_{i=s+1}^{j} v_{i} \phi_{j-i} \\
& =: \lambda^{t-s} x_{s}+\Delta_{s, t}+x_{s, t}^{\prime},
\end{aligned}
$$

where

$x_{s, t}^{\prime}=\sum_{j=1}^{t-s} \lambda^{t-j-s} \sum_{i=1}^{j} v_{i+s} \phi_{j-i}=\sum_{i=s+1}^{t} v_{i} \sum_{j=0}^{t-i} \lambda^{t-j-i} \phi_{j}$.

Write $d_{s, t}^{2}=\sum_{i=s+1}^{t} \lambda^{2(t-i)}\left(\sum_{j=0}^{t-i} \lambda^{-j} \phi_{j}\right)^{2}=E\left(x_{s, t}^{\prime}\right)^{2}$. Recall $\lim _{n \rightarrow \infty} \lambda^{n}=e^{\tau}$ and $\lim _{n \rightarrow \infty} \lambda^{m}=1$ for any fixed $m$. Routine calculations show that, whenever $n$ is sufficiently large,

$e^{-|\tau|} / 2 \leq \lambda^{k} \leq 2 e^{|\tau|}, \quad$ for all $-n \leq k \leq n$

and there exist $\gamma_{1}, \gamma_{0}>0$ such that

$\gamma_{0} \leq \inf _{n \geq k \geq m}\left|\sum_{j=0}^{k} \lambda^{-j} \phi_{j}\right| \leq \gamma_{1}$,

whenever $n, m$ are sufficiently large. By virtue of (A.2) and (A.3), it is readily seen that $d_{s, t} \neq 0$ for all $0 \leq s<t \leq n$ because $\phi=\sum_{j=0}^{\infty} \phi_{j} \neq 0$ and $C_{1}(t-s) \leq d_{s, t}^{2} \leq C_{2}(t-s)$. Consequently,

$\frac{1}{\sqrt{t-s}} x_{s, t}^{\prime}$ has a density $h_{s, t}(x)$,

which is uniformly bounded by a constant $C_{0}$ and $\int_{-\infty}^{\infty}(1+|u|)\left|\varphi_{s, t}(u)\right| d u<\infty$ uniformly for $0 \leq s<t \leq n$, where $\varphi_{s, t}(u)=E e^{i u x_{s, t}^{\prime} / \sqrt{t-s}}$, due to $\int(1+|u|)\left|E e^{i u v_{0}}\right| d t<\infty$. See the proof of Corollary 2.2 in Wang and Phillips (2009a) and/or (7.14) and Proposition 7.2 (p. 1934) of Wang and Phillips (2009b) with a minor modification. Hence, conditional on $\mathcal{F}_{k}=\sigma\left(v_{j},-\infty<j \leq k\right)$,

$\left(x_{t}-x_{s}\right) / \sqrt{t-s}$ has a density $h_{s, t}^{*}(x) \equiv h_{s, t}\left(x-x_{s, t}^{*} / \sqrt{t-s}\right)$, 
where $x_{s, t}^{*}=\left(\lambda^{t-s}-1\right) x_{s}+\Delta_{s, t}$, satisfying, for any $u \in \mathbb{R}$,

$$
\begin{aligned}
& \sup _{x}\left|h_{s, t}^{*}(x+u)-h_{s, t}^{*}(x)\right| \leq \sup _{x}\left|h_{s, t}(x+u)-h_{s, t}(x)\right| \\
& \quad \leq C\left|\int_{-\infty}^{\infty}\left(e^{-i v(x+u)}-e^{-i v x}\right) \varphi_{s, t}(v) d v\right| \\
& \quad \leq C \min \{|u|, 1\} \int_{-\infty}^{\infty}(1+|v|)\left|\varphi_{s, t}(v)\right| d v \leq C_{1} \min \{|u|, 1\},
\end{aligned}
$$

where we have used the inversion formula of a characteristic function in the calculation above.

We also have the following representation for $x_{t}$ :

$$
\begin{aligned}
x_{t} & =\sum_{j=1}^{t} \lambda^{t-j} \xi_{j}=\sum_{j=1}^{t} \lambda^{t-j}\left(\sum_{i=0}^{j-1}+\sum_{i=j}^{\infty}\right) \phi_{i} v_{j-i} \\
& =\sum_{i=0}^{t-1} \phi_{i} \lambda^{t-i} \sum_{j=1}^{t} \lambda^{-j} v_{j}-\sum_{i=0}^{t-1} \phi_{i} \lambda^{t-i} \sum_{j=t-i+1}^{t} \lambda^{-j} v_{j}+\sum_{j=1}^{t} \lambda^{t-j} \sum_{i=0}^{\infty} \phi_{i+j} v_{-i} \\
& =a_{t} x_{t}^{\prime}-x_{t}^{\prime \prime}+x_{t}^{\prime \prime \prime}, \quad \text { say, }
\end{aligned}
$$

where $a_{t}=\sum_{i=0}^{t-1} \phi_{i} \lambda^{-i}, x_{t}^{\prime}=\sum_{j=1}^{t} \lambda^{t-j} v_{j}$, and

$\left|x_{t}^{\prime \prime}\right|+\left|x_{t}^{\prime \prime \prime}\right| \leq C_{0} t^{1 /(2+\delta)}, \quad$ a.s.

for some constant $C_{0}>0$. Indeed, using (A.2) and the strong law, we obtain that, for some constant $C_{0}>0$,

$$
\begin{aligned}
\left|x_{t}^{\prime \prime}\right| & \leq 2 e^{|\tau|} \sum_{i=0}^{t-1}\left|\phi_{i}\right| \sum_{j=t-i+1}^{t}\left|v_{j}\right| \leq 2 e^{|\tau|} \max _{1 \leq j \leq t}\left|v_{j}\right| \sum_{i=0}^{t-1} i\left|\phi_{i}\right| \\
& \leq C t^{1 /(2+\delta)}\left(\frac{1}{t} \sum_{j=1}^{t}\left|v_{j}\right|^{2+\delta}\right)^{1 /(2+\delta)} \leq C_{0} t^{1 /(2+\delta)}, \quad \text { a.s. },
\end{aligned}
$$

since $E\left|v_{1}\right|^{2+\delta}<\infty$ and $\sum_{i=0}^{\infty} i\left|\phi_{i}\right|<\infty$. Note that

$$
\begin{aligned}
\sum_{j=1}^{\infty} j^{-1 /(2+\delta)} E\left|\sum_{i=0}^{\infty} \phi_{i+j} \nu_{-i}\right| & \leq \sum_{j=1}^{\infty} j^{-1 /(2+\delta)}\left(\sum_{i=j}^{\infty} \phi_{i}^{2}\right)^{1 / 2} \\
& \leq C \sum_{j=1}^{\infty} j^{-1-1 /(2+\delta)}\left(\sum_{i=j}^{\infty} i\left|\phi_{i}\right|\right)^{1 / 2}<\infty
\end{aligned}
$$

which yields that $\sum_{j=1}^{\infty} j^{-1 /(2+\delta)}\left|\sum_{i=0}^{\infty} \phi_{i+j} v_{-i}\right|<\infty$, a.s. It follows from (A.2) again and the Kronecker lemma that

$$
\left|x_{t}^{\prime \prime \prime}\right| \leq C \sum_{j=1}^{t}\left|\sum_{i=0}^{\infty} \phi_{i+j} v_{-i}\right|=o\left(t^{1 /(2+\delta)}\right), \quad \text { a.s. }
$$

This proves (A.6). 
We are now ready to provide several preliminary lemmas.

LEMMA A.1. Suppose that $p(x)$ satisfies $\int|p(x)| d x<\infty$ and Assumption 2.1 holds. Then, for any $h \rightarrow 0$ and all $0 \leq s<t \leq n$, we have

$$
\begin{aligned}
E\left(\left|p\left(x_{t} / h\right)\right| \mid \mathcal{F}_{s}\right) & \leq \frac{C_{0} h}{\sqrt{t-s}} \int_{-\infty}^{\infty}\left|p\left(x+x_{s} / h\right)\right| d x \\
& =\frac{C_{0} h}{\sqrt{t-s}} \int_{-\infty}^{\infty}|p(x)| d x, \quad \text { a.s. }
\end{aligned}
$$

where $\mathcal{F}_{s}=\sigma\left\{v_{s}, v_{s-1}, \ldots\right\}$.

Proof. Recall (A.1), (A.4), and the independence of $v_{k}$. The result (A.8) follows from a routine calculation and hence the details are omitted.

LEMMA A.2. Suppose that $p(x)$ satisfies $\int\left[|p(x)|+p^{2}(x)\right] d x<\infty$ and $\int p(x) d x \neq 0$ and that Assumption 2.1 holds. Then, for any $h \rightarrow 0$ and $n h^{2} \rightarrow \infty$,

$\frac{\phi}{\sqrt{n} h} \sum_{t=1}^{n} p\left[\left(x_{t}-x\right) / h\right] \rightarrow D \int_{-\infty}^{\infty} p(x) d x L_{G}(1,0)$,

where $G(t)=W(t)+\tau \int_{0}^{t} e^{\tau(t-s)} W(s) d s$ with $W(s)$ being a standard Brownian motion and $L_{G}(r, x)$ is a local time of the Gaussian process $G(t)$.

Proof. This is a corollary of Theorem 3.1 of Wang and Phillips (2009a). The inspection of the conditions is similar to Proposition 7.2 of Wang and Phillips (2009b). We omit the details.

LEMMA A.3. Suppose that Assumptions 2.1, 2.2, and 2.3(a) hold. Then, for any $h \rightarrow 0$ and $n h^{2} \rightarrow \infty$,

$\sum_{t=1}^{n} u_{t} Z_{n t} \rightarrow{ }_{D} N\left(0, \sigma_{1}^{2}\right)$

where $Z_{n t}=K\left[\left(x_{t}-x\right) / h\right] /\left(\sum_{t=1}^{n} K\left[\left(x_{t}-x\right) / h\right]\right)^{1 / 2}$ and $\sigma_{1}^{2}=\left(1-\rho^{2}\right)^{-1} \sigma^{2} \int_{-\infty}^{\infty} K^{2}(x) d x$.

Proof. For notational convenience, we assume that $\sigma^{2}=1$ in the following proof. Note that $u_{t}=\sum_{k=1}^{t} \rho^{t-k} \epsilon_{k}$. We have $\sum_{t=1}^{n} u_{t} Z_{n t}=\sum_{k=1}^{n} \epsilon_{k} Z_{n k}^{*}$, where $Z_{n k}^{*}=$ $\sum_{t=k}^{n} \rho^{t-k} Z_{n t}$. We first claim that

$$
\begin{aligned}
& \sum_{k=1}^{n} Z_{n k}^{2} \rightarrow P \int_{-\infty}^{\infty} K^{2}(x) d x, \\
& \sum_{k=1}^{n} Z_{n k}^{* 2} \rightarrow_{P}\left(1-\rho^{2}\right)^{-1} \int_{-\infty}^{\infty} K^{2}(x) d x .
\end{aligned}
$$


The proof of (A.10) is simple by applying Lemma A.2. To see (A.11), note that

$$
\begin{aligned}
\sum_{k=1}^{n} Z_{n k}^{* 2} & =\Lambda_{n}^{-1} \sum_{k=1}^{n}\left(\sum_{t=k}^{n} \rho^{t-k} K\left[\left(x_{t}-x\right) / h\right]\right)^{2} \\
& =\Lambda_{n}^{-1} \sum_{k=1}^{n} \sum_{t=k}^{n} \rho^{2(t-k)} K^{2}\left[\left(x_{t}-x\right) / h\right]+\Lambda_{n}^{-1} \Gamma_{1 n} \\
& =\left(1-\rho^{2}\right)^{-1} \sum_{k=1}^{n} Z_{n k}^{2}+\Lambda_{n}^{-1}\left(\Gamma_{1 n}-\Gamma_{2 n}\right) \\
& =\left(1-\rho^{2}\right)^{-1} \int_{-\infty}^{\infty} K^{2}(x) d x+\Lambda_{n}^{-1}\left(\Gamma_{1 n}-\Gamma_{2 n}\right)+o_{P}(1)
\end{aligned}
$$

by (A.10), where $\Lambda_{n}=\sum_{t=1}^{n} K\left[\left(x_{t}-x\right) / h\right]$,

$$
\begin{aligned}
& \Gamma_{1 n}=2 \sum_{k=1}^{n} \sum_{k \leq s<t \leq n} \rho^{s-k} \rho^{t-k} K\left[\left(x_{s}-x\right) / h\right] K\left[\left(x_{t}-x\right) / h\right], \\
& \Gamma_{2 n}=\left(1-\rho^{2}\right)^{-1} \sum_{t=1}^{n} K^{2}\left[\left(x_{t}-x\right) / h\right] \rho^{2 t} .
\end{aligned}
$$

Note that $\Lambda_{n} /(\sqrt{n} h) \rightarrow{ }_{D} \phi^{-1} L_{G}(1,0)$ by Lemma A.2. The result (A.11) will follow if we prove

$\Gamma_{1 n}+\Gamma_{2 n}=o_{P}\left[\left(n h^{2}\right)^{1 / 2}\right]$.

Recalling that $K(x) \leq C$ and $|\rho|<1$, it is readily seen that $\Gamma_{2 n} \leq C$. On the other hand, by applying Lemma A.1, for any $t>s$, we have

$$
\begin{aligned}
E\left\{K\left[\left(x_{s}-x\right) / h\right] K\left[\left(x_{t}-x\right) / h\right]\right\} & \leq E\left[K\left[\left(x_{s}-x\right) / h\right] E\left\{K\left[\left(x_{t}-x\right) / h\right] \mid \mathcal{F}_{s}\right\}\right] \\
& \leq \frac{C h}{\sqrt{t-s}} \frac{h}{\sqrt{s}} .
\end{aligned}
$$

It follows that

$$
\begin{aligned}
E \Gamma_{1 n} & \leq C h^{2} \sum_{k=1}^{n} \sum_{k \leq s<t \leq n} \rho^{s-k} \rho^{t-k} \frac{1}{\sqrt{t-s}} \frac{1}{\sqrt{s}} \\
& \leq C_{1} h^{2} \sum_{k=1}^{n} \frac{1}{\sqrt{k}} \leq C h^{2} \sqrt{n},
\end{aligned}
$$

which implies that $\Gamma_{1 n}=O_{P}\left(h^{2} \sqrt{n}\right)$. Hence, (A.13) follows due to $h \rightarrow 0$ and $n h^{2} \rightarrow \infty$. This also completes the proof of (A.11).

We now turn to the proof of (A.9). Since, given $\left\{x_{1}, x_{2}, \ldots, x_{n}\right\}$, the sequence $\left(Z_{n k}^{*} \epsilon_{k}\right.$, $k=1,2, \ldots, n)$ still forms a martingale difference by Assumption 2.2, it follows from Theorem 3.9 [(3.75)] in Hall and Heyde (1980) with $\delta=q / 2-1$ that $\sup _{x}\left|P\left(\sum_{t=1}^{n} \epsilon_{t} Z_{n t}^{*} \leq x \sigma_{1} \mid x_{1}, x_{2}, \ldots, x_{n}\right)-\Phi(x)\right| \leq A(\delta) \mathcal{L}_{n}^{1 /(1+q)}, \quad$ a.s., 
where $A(\delta)$ is a constant depending only on $q>2$ and

$$
\begin{aligned}
\mathcal{L}_{n}= & \frac{1}{\sigma_{1}^{q}} \sum_{k=1}^{n}\left|Z_{n k}^{*}\right|^{q} E\left(\left|\epsilon_{k}\right|^{q} \mid x_{1}, \ldots, x_{n}\right) \\
& +E\left\{\left|\frac{1}{\sigma_{1}^{2}} \sum_{k=1}^{n} Z_{n k}^{* 2}\left[E\left(\epsilon_{k}^{2} \mid \mathcal{F}_{k-1}\right)-1\right]\right|^{q / 2} \mid x_{1}, \ldots, x_{n}\right\} .
\end{aligned}
$$

Recall that $K(x)$ is uniformly bounded and that

$$
\max _{1 \leq k \leq n}\left|Z_{n k}^{*}\right| \leq C \max _{1 \leq k \leq n}\left|Z_{n k}\right| \leq C /\left(\sum_{t=1}^{n} K\left[\left(x_{t}-x\right) / h\right]\right)^{1 / 2}=o_{P}(1),
$$

by Assumption 2.3 and Lemma A.2. Routine calculations, together with (A.11), show that $\mathcal{L}_{n}=o_{P}(1)$,

since $q>2$. Therefore the dominated convergence theorem yields that

$$
\begin{aligned}
\sup _{x}\left|P\left(\sum_{t=1}^{n} u_{t} Z_{n t} \leq x \sigma_{1}\right)-\Phi(x)\right| \\
\leq E\left[\sup _{x}\left|P\left(\sum_{t=1}^{n} \epsilon_{t} Z_{n t}^{*} \leq x \sigma_{1} \mid x_{1}, x_{2}, \ldots, x_{n}\right)-\Phi(x)\right|\right] \rightarrow 0 .
\end{aligned}
$$

This completes the proof of Lemma A.3.

LEMMA A.4. Suppose that Assumptions 2.3(a) and 2.4(a) hold. Then, for any $x \in \mathbb{R}$, we have

$\left|\Lambda_{n}(x)-m(x)\right| \leq C\left(1+|x|^{\alpha}\right) h^{\beta}$,

where $\Lambda_{n}(x)=\frac{\sum_{t=1}^{n} m\left(x_{t}\right) K\left[\left(x_{t}-x\right) / h\right]}{\sum_{t=1}^{n} K\left[\left(x_{t}-x\right) / h\right]}$. If in addition Assumption 2.4(b) holds, we have

$\left|\Lambda_{n}(x)-m(x)-\frac{h^{p}(p)(x)}{p !} \int_{-\infty}^{\infty} y^{p} K(y) d y\right|=o_{P}\left[\left(n h^{2}\right)^{-1 / 4}\right]$,

whenever $n h^{2} \rightarrow \infty$ and $n h^{2+4 p}=O(1)$, for any fixed $x$.

Proof. By Assumption 2.4(a) and the fact that $K(x)$ has a compact support, the result (A.14) is simple. The proof of (A.15) is the same as in the proof of Theorem 2.2 in Wang and Phillips (2011). We omit the details.

LEMMA A.5. Suppose that Assumptions 3.1-3.4 hold. Then, for any $l>0$, we have

$$
I_{n}:=\sup _{t \in R} \sup _{s:|s-t| \leq \epsilon_{n}}\left|\frac{c_{n}}{n} \sum_{k=1}^{n} f_{t, s}\left(x_{k, n}\right)\right|=o\left(\log ^{-l} n\right), \quad \text { a.s., }
$$

where $\epsilon_{n} \leq c_{n} n^{-l_{1}}$ for some $l_{1}>0$ and $f_{t, s}(x)=g\left(c_{n} x+t\right)-g\left(c_{n} x+s\right)$.

Proof. See Lemma 3.5 of Liu, Chan, and Wang (2014). 


\section{A.2. Proofs of Theorems 2.1 and 2.2}

We only prove Theorem 2.2. Using Lemmas A.3 and A.4, the proof of Theorem 2.1 is standard (see, e.g., Wang and Phillips, 2011), and hence the details are omitted.

Start with (2.6). Recall that $\hat{u}_{t}=y_{t}-\hat{m}\left(x_{t}\right)=u_{t}+m\left(x_{t}\right)-\hat{m}\left(x_{t}\right)$. Simple calculations show that

$$
\begin{aligned}
\hat{\rho}-\rho & =\frac{\sum_{s=2}^{n}\left(\widehat{u}_{s}-\rho \widehat{u}_{s-1}\right) \widehat{u}_{s-1}}{\sum_{s=2}^{n} \widehat{u}_{s-1}^{2}} \\
& =\frac{\sum_{s=2}^{n} \epsilon_{s} \widehat{u}_{s-1}}{\sum_{s=2}^{n} \widehat{u}_{s-1}^{2}}+\frac{\sum_{s=2}^{n} \widehat{u}_{s-1}\left[m\left(x_{s}\right)-\hat{m}\left(x_{s}\right)+\rho\left\{\hat{m}\left(x_{s-1}\right)-m\left(x_{s-1}\right)\right\}\right]}{\sum_{s=2}^{n} \widehat{u}_{s-1}^{2}} \\
& =: R_{1 n}+R_{2 n} .
\end{aligned}
$$

As $V_{n}=\frac{1}{n} \sum_{t=1}^{n}\left[\hat{m}\left(x_{t}\right)-m\left(x_{t}\right)\right]^{2}=O_{P}\left\{n^{\alpha} h^{2 \beta}+\left(n h^{2}\right)^{-1 / 2}\right\}$ by (3.5) of Theorem 3.2, it follows from $\frac{1}{n} \sum_{t=1}^{n} u_{t}^{2} \rightarrow\left(1-\rho^{2}\right)^{-1} \sigma^{2}$, a.s., that $\frac{1}{n} \sum_{s=2}^{n} \widehat{u}_{s-1}^{2} \rightarrow P\left(1-\rho^{2}\right)^{-1} \sigma^{2}$, whenever $n^{\alpha} h^{2 \beta} \rightarrow 0$ and $n h^{2} \rightarrow \infty$. This, together with Hölder's inequality, yields that

$$
\left|R_{2 n}\right| \leq 2\left(1+\rho^{2}\right)^{1 / 2} V_{n}^{1 / 2} /\left(\sum_{s=2}^{n} \widehat{u}_{s-1}^{2}\right)^{1 / 2}=O_{P}\left\{n^{\alpha / 2} h^{\beta}+\left(n h^{2}\right)^{-1 / 4}\right\} .
$$

On the other hand, by recalling Assumption 2.2, it is readily seen that $R_{1 n}=O_{P}\left(n^{-1 / 2}\right)$. Taking these facts into (A.17), we obtain (2.6).

We next prove (2.7). We may write

$$
\begin{aligned}
\hat{m}_{1}(x)-m(x)= & \frac{\widehat{\rho} \sum_{t=1}^{n}\left[\hat{m}_{1}\left(x_{t-1}\right)-m\left(x_{t-1}\right)\right] K\left[\left(x_{t}-x\right) / h\right]}{\sum_{t=1}^{n} K\left[\left(x_{t}-x\right) / h\right]} \\
& +\frac{(\rho-\widehat{\rho}) \sum_{t=1}^{n} u_{t-1} K\left[\left(x_{t}-x\right) / h\right]}{\sum_{t=1}^{n} K\left[\left(x_{t}-x\right) / h\right]} \\
& +\frac{\sum_{t=1}^{n}\left[m\left(x_{t}\right)-m(x)\right] K\left[\left(x_{t}-x\right) / h\right]}{\sum_{t=1}^{n} K\left[\left(x_{t}-x\right) / h\right]}+\frac{\sum_{t=1}^{n} \epsilon_{t} K\left[\left(x_{t}-x\right) / h\right]}{\sum_{t=1}^{n} K\left[\left(x_{t}-x\right) / h\right]} \\
:= & \widehat{\rho} I_{1 n}+(\rho-\widehat{\rho}) I_{2 n}+I_{3 n}+I_{4 n} .
\end{aligned}
$$

Furthermore, we may divide $I_{1 n}$ into

$$
\begin{aligned}
I_{1 n}= & \frac{1}{\sum_{t=1}^{n} K\left[\left(x_{t}-x\right) / h\right]} \\
& \times\left\{\sum_{t=1}^{n} K\left[\left(x_{t}-x\right) / h\right] \frac{\sum_{s=1}^{n}\left[m\left(x_{s}\right)-m\left(x_{t-1}\right)\right] K\left[\left(x_{s}-x_{t-1}\right) / h\right]}{\sum_{s=1}^{n} K\left[\left(x_{s}-x_{t-1}\right) / h\right]}\right. \\
& \left.\quad+\sum_{t=1}^{n} K\left[\left(x_{t}-x\right) / h\right] \frac{\sum_{s=1}^{n} u_{s} K\left[\left(x_{s}-x_{t-1}\right) / h\right]}{\sum_{s=1}^{n} K\left[\left(x_{s}-x_{t-1}\right) / h\right]}\right\} \\
:= & I_{1 n 1}+I_{\ln 2 .}
\end{aligned}
$$


Using Lemma A.3 and (2.6), we have

$(\rho-\widehat{\rho})\left(\sum_{t=1}^{n} K\left[\left(x_{t}-x\right) / h\right]\right)^{1 / 2} I_{2 n} \rightarrow{ }_{P} 0$

and (with $\rho=0$ in Lemma A.3)

$\left(\sum_{t=1}^{n} K\left[\left(x_{t}-x\right) / h\right]\right)^{1 / 2} I_{4 n} \rightarrow{ }_{D} N\left(0, \sigma_{2}^{2}\right)$.

Using (A.14) and $\frac{1}{\sqrt{n} h} \sum_{t=1}^{n} K\left[\left(x_{t}-x\right) / h\right] \rightarrow{ }_{D} \phi^{-1} L_{G}(1,0)$, we have

$$
\begin{aligned}
& \left(\sum_{t=1}^{n} K\left[\left(x_{t}-x\right) / h\right]\right)^{1 / 2}\left|I_{3 n}\right| \\
& \quad \leq C\left(n h^{2+4 \beta}\right)^{1 / 4}\left(\frac{1}{\sqrt{n} h} \sum_{t=1}^{n} K\left[\left(x_{t}-x\right) / h\right]\right)^{1 / 2}=o_{P}(1) .
\end{aligned}
$$

Similarly, by recalling that $K$ has a compact support, we obtain

$$
\begin{aligned}
& \left(\sum_{t=1}^{n} K\left[\left(x_{t}-x\right) / h\right]\right)^{1 / 2}\left|I_{1 n 1}\right| \\
& \quad \leq C h^{\beta}\left(\sum_{t=1}^{n} K\left[\left(x_{t}-x\right) / h\right]\right)^{-1 / 2} \sum_{t=1}^{n}\left(1+\left|x_{t}\right|^{\alpha}\right) K\left[\left(x_{t}-x\right) / h\right] \\
& \quad \leq C\left(n h^{2+4 \beta}\right)^{1 / 4}\left(\frac{1}{\sqrt{n} h} \sum_{t=1}^{n} K\left[\left(x_{t}-x\right) / h\right]\right)^{1 / 2}=o_{P}(1) .
\end{aligned}
$$

Combining all the above facts, to prove (2.7), it suffices to show that

$I_{1 n 2}=o_{P}\left[\left(n h^{2}\right)^{-1 / 4}\right]$.

To this end, for each fixed $x$, write

$\Omega_{1 n}=\left\{\omega: \sum_{s=1}^{n} K\left[\left(x_{s}-x\right) / h\right] \geq \sqrt{n} h \delta_{n}\right\}$,
$\Omega_{2 n}=\left\{\omega: \inf _{t=1,2, \ldots, n} \sum_{s=1}^{n} K\left[\left(x_{s}-x_{t}\right) / h\right] \geq \sqrt{n} h \delta_{n}\right\}$,

where $\delta_{n} \downarrow 0$ is chosen later and $\omega$ denotes the sample points. As $P\left(\bar{\Omega}_{1 n} \cup \bar{\Omega}_{2 n}\right) \rightarrow 0$ by (3.4) and Lemma A.2, the result (A.18) will follow if we prove

$I_{\Omega_{1 n}}(\omega) I_{\Omega_{2 n}}(\omega) I_{1 n 2}=o_{P}\left[\left(n h^{2}\right)^{-1 / 4}\right]$,

where $I_{A}(\omega)$ denotes the indicator function. 
Recall that $u_{s}=\sum_{k=1}^{s} \rho^{s-k} \epsilon_{k}$. Then, $I_{1 n 2}$ can be rewritten as

$$
\begin{aligned}
I_{1 n 2} & =\frac{1}{\sum_{t=1}^{n} K\left[\left(x_{t}-x\right) / h\right]} \sum_{s=1}^{n} u_{s} J_{n s} \\
& =\frac{1}{\sum_{t=1}^{n} K\left[\left(x_{t}-x\right) / h\right]} \sum_{k=1}^{n} \epsilon_{k} \sum_{s=k}^{n} \rho^{s-k} J_{n s},
\end{aligned}
$$

where

$$
J_{n s}=\sum_{t=1}^{n} \frac{K\left[\left(x_{s}-x_{t-1}\right) / h\right] K\left[\left(x_{t}-x\right) / h\right]}{\sum_{s=1}^{n} K\left[\left(x_{s}-x_{t-1}\right) / h\right]} .
$$

It follows easily from the conditional arguments and Hölder's inequality that

$$
\begin{aligned}
E\left[I_{\Omega_{1 n}}(\omega) I_{\Omega_{2 n}}(\omega) I_{2 n 2}\right]^{2} & \leq \frac{C}{\left(n h^{2} \delta_{n}\right)^{2}} \sum_{k=1}^{n} E\left(\sum_{s=k}^{n} \rho^{s-k} J_{n s}^{*}\right)^{2} \\
& \leq \frac{C}{\left(n h^{2} \delta_{n}\right)^{2}} \sum_{k=1}^{n} \sum_{s=k}^{n} \rho^{s-k} \sum_{s=k}^{n} \rho^{s-k} E J_{n s}^{* 2} \\
& \leq \frac{n C}{\left(n h^{2} \delta_{n}\right)^{2}} \max _{1 \leq s \leq n} E J_{n s}^{* 2},
\end{aligned}
$$

where

$$
J_{n s}^{*}=\sum_{t=1}^{n} K\left[\left(x_{s}-x_{t-1}\right) / h\right] K\left[\left(x_{t}-x\right) / h\right] .
$$

Simple calculations show that, by letting $\sum_{k=i}^{j}=0$ if $j<i$,

$$
\begin{aligned}
E J_{n s}^{* 2}= & E\left(\sum_{t=1}^{n} K\left[\left(x_{s}-x_{t-1}\right) / h\right] K\left[\left(x_{t}-x\right) / h\right]\right)^{2} \\
\leq & 4 E\left\{\sum_{t=1}^{s-1} K\left[\left(x_{s}-x_{t-1}\right) / h\right] K\left[\left(x_{t}-x\right) / h\right]\right\}^{2} \\
& +4 E\left\{\sum_{t=s+1}^{n} K\left[\left(x_{s}-x_{t-1}\right) / h\right] K\left[\left(x_{t}-x\right) / h\right]\right\}^{2} \\
& +4 E K^{2}\left[\left(x_{s}-x_{s-1}\right) / h\right] K^{2}\left[\left(x_{s}-x\right) / h\right] \\
:= & T_{1 n}+T_{2 n}+T_{3 n} .
\end{aligned}
$$

Assume $t_{1}<t_{2} \leq s-1$. Recall that $K(x)$ has a compact support. It follows from Lemma A.1 with $p(x)=K\left(x-x_{t_{1}-1} / h\right) K\left(x-x_{t_{2}-1} / h\right)$ that 


$$
\begin{aligned}
& E\left\{K\left[\left(x_{s}-x_{t_{1}-1}\right) / h\right] K\left[\left(x_{s}-x_{t_{2}-1}\right) / h\right] \mid \mathcal{F}_{t_{2}}\right\} \\
& \quad \leq \frac{C h}{\sqrt{s-t_{2}}} \int_{-\infty}^{\infty} K\left[y+\left(x_{t_{2}}-x_{t_{1}-1}\right) / h\right] K\left[y+\left(x_{t_{2}}-x_{t_{2}-1}\right) / h\right] d y \\
& \quad \leq \frac{C h}{\sqrt{s-t_{2}}} \int_{-\infty}^{\infty} K(y) K\left[y+\left(x_{t_{2}-1}-x_{t_{1}-1}\right) / h\right] d y, \quad \text { a.s. }
\end{aligned}
$$

This, together with the repeatedly similar utilization of Lemma A.1, yields that, for $t_{1}<t_{2} \leq s-1$,

$$
\begin{aligned}
\Psi_{s, t_{1}, t_{2}}: & =E\left\{K\left[\left(x_{s}-x_{t_{1}-1}\right) / h\right] K\left[\left(x_{s}-x_{t_{2}-1}\right) / h\right] K\left[\left(x_{t_{1}}-x\right) / h\right] K\left[\left(x_{t_{2}}-x\right) / h\right]\right\} \\
\leq & \frac{C h}{\sqrt{s-t_{2}}} \int_{-\infty}^{\infty} E\left\{K\left[y+\left(x_{t_{2}-1}-x_{t_{1}-1}\right) / h\right]\right. \\
& \left.\quad \times K\left[\left(x_{t_{1}}-x\right) / h\right] K\left[\left(x_{t_{2}}-x\right) / h\right]\right\} K(y) d y \\
\leq & \frac{C h^{2}}{\sqrt{s-t_{2}}} \int_{-\infty}^{\infty} E\left\{K\left[y+\left(x_{t_{2}-1}-x_{t_{1}-1}\right) / h\right] K\left[\left(x_{t_{1}}-x\right) / h\right]\right\} K(y) d y \\
\leq & \frac{C h^{4}}{\sqrt{s-t_{2}}} \frac{1}{\sqrt{t_{2}-t_{1}}} \frac{1}{\sqrt{t_{1}}} .
\end{aligned}
$$

Similarly, for $t_{1}=t_{2}<s-1$,

$$
\Psi_{s, t_{1}, t_{1}}=E\left\{K^{2}\left[\left(x_{s}-x_{t_{1}-1}\right) / h\right] K^{2}\left[\left(x_{t_{1}}-x\right) / h\right]\right\} \leq \frac{C h^{2}}{\sqrt{s-t_{1}}} \frac{1}{\sqrt{t_{1}}} .
$$

We now obtain, for any $1 \leq s \leq n$,

$$
\begin{aligned}
T_{1 n} & =\sum_{t_{1}=1}^{s-2} \Psi_{s, t_{1}, t_{1}}+2 \sum_{1 \leq t_{1}<t_{2} \leq s-2} \Psi_{s, t_{1}, t_{2}} \\
& \leq \sum_{t_{1}=1}^{s-2} \frac{C h^{2}}{\sqrt{s-t_{1}}} \frac{1}{\sqrt{t_{1}}}+\sum_{1 \leq t_{1}<t_{2} \leq s-2} \frac{C h^{4}}{\sqrt{s-t_{2}}} \frac{1}{\sqrt{t_{2}-t_{1}}} \frac{1}{\sqrt{t_{1}}} \\
& \leq C h^{2}\left(1+\sqrt{n} h^{2}\right) .
\end{aligned}
$$

Similarly we may prove

$T_{2 n}+T_{3 n} \leq C h^{2}\left(1+\sqrt{n} h^{2}\right)$.

Combining all these estimates, it follows from (A.20) that

$E\left[I_{\Omega_{1 n}}(\omega) I_{\Omega_{2 n}}(\omega) I_{2 n 2}\right]^{2} \leq C \delta_{n}^{-2}\left[\left(n h^{2}\right)^{-1}+n^{-1 / 2}\right]=o\left[\left(n h^{2}\right)^{-1 / 2}\right]$,

by choosing $\delta_{n}=\min \left\{\left(n h^{2}\right)^{1 / 8}, h^{-1 / 4}\right\} \rightarrow \infty$, whenever $h \rightarrow 0$ and $n h^{2} \rightarrow \infty$. This proves (A.18) and also completes the proof of Theorem 2.2.

\section{A.3. Proof of Theorem 3.1}

The idea for the proof of (3.3) is similar to that of Liu et al. (2014), but there are some essential differences in details. We restate here for the convenience of reading. Without 
loss of generality, we assume $\tau=\int g(x) d x=1$. Define $\bar{g}(x)=g(x) I\left\{|x| \leq n^{\zeta} / 2\right\}$, where $0<\zeta<1-\delta_{0} / \gamma$ is small enough such that $n^{\zeta} / c_{n} \leq \min \left\{n^{-\delta_{0}}, n^{-\epsilon_{0} / 2}\right\}$, where $\gamma$ and $\delta_{0}$ are given in Assumptions 3.1 and 3.2, respectively. Further let $\epsilon=n^{-\alpha}$ with $0<\alpha<\delta_{0} / 2$ and define a triangular function

$g_{\epsilon}(y)= \begin{cases}0, & |y|>\epsilon, \\ \frac{y+\epsilon}{\epsilon^{2}}, & -\epsilon \leq y \leq 0, \\ \frac{\epsilon-y}{\epsilon^{2}}, & 0 \leq y \leq \epsilon,\end{cases}$

It suffices to show that:

$$
\begin{aligned}
\Phi_{1 n} & :=\max _{1 \leq k \leq n}\left|\frac{c_{n}}{n} \sum_{j=1}^{n}\left\{g\left[c_{n}\left(x_{j, n}-x_{k, n}\right)\right]-\bar{g}\left[c_{n}\left(x_{j, n}-x_{k, n}\right)\right]\right\}\right| \\
& =o_{a . s .}\left(\log ^{-l} n\right), \\
\Phi_{2 n} & :=\max _{1 \leq k \leq n}\left|\frac{c_{n}}{n} \sum_{j=1}^{n} \bar{g}\left[c_{n}\left(x_{j, n}-x_{k, n}\right)\right]-\frac{1}{n} \sum_{j=1}^{n} g_{\epsilon}\left(x_{j, n}-x_{k, n}\right)\right| \\
& =o_{a . s .}\left(\log ^{-l} n\right), \\
\Phi_{3 n} & :=\sup _{0 \leq t \leq 1}\left|\frac{1}{n} \sum_{j=1}^{n} g_{\epsilon}\left(x_{j, n}-x_{[n t], n}\right)-L_{n t}\right|=o_{P}\left(\log ^{-l} n\right) .
\end{aligned}
$$

The proof of (A.21) is simple. Indeed, by recalling $\sup _{x}|x|^{\gamma}|g(x)|<\infty$, it follows that

$\Phi_{1 n} \leq c_{n} \sup _{|x| \geq n^{\zeta} / 2}|g(x)| I\left\{|x|>n^{\zeta} / 2\right\} \leq C n^{-\zeta \gamma} c_{n}=o\left(\log ^{-l} n\right)$

as $n^{\zeta} / c_{n} \leq n^{-\delta_{0}}$ and $\gamma>\delta_{0} /(1-\zeta)$

We next prove (A.23). Recalling $\int_{-\infty}^{\infty} g_{\epsilon}(y) d y=1$, it follows from the definition of occupation time and (3.2) in Assumption 3.2 that

$$
\begin{aligned}
& \left|\int_{0}^{1} g_{\epsilon}[G(s)-G(t)] d s-L_{G}(1, G(t))\right| \\
& \quad=\left|\int_{-\infty}^{\infty} g_{\epsilon}[y-G(t)] L_{G}(1, y) d y-L_{G}(1, G(t))\right| \\
& \quad \leq \int_{-\infty}^{\infty} g_{\epsilon}(y)\left|L_{G}(1, y+G(t))-L_{G}(1, G(t))\right| d y \\
& \quad \leq C \epsilon^{\xi} \text { a.s. }
\end{aligned}
$$

for some $\xi>0$, uniformly for $t \in[0,1]$. Hence, by noting

$$
\int_{0}^{1} g_{\epsilon}\left[G_{n}(s)-G_{n}(t)\right] d s-L_{n t}={ }_{D} \int_{0}^{1} g_{\epsilon}[G(s)-G(t)] d s-L_{G}(1, G(t)),
$$


due to $\left\{G_{n}(t) ; 0 \leq t \leq 1\right\}={ }_{D}\{G(t) ; 0 \leq t \leq 1\}, n \geq 1$, we have

$$
\begin{aligned}
& P\left(\left|\int_{0}^{1} g_{\epsilon}\left[G_{n}(s)-G_{n}(t)\right] d s-L_{n t}\right| \geq \log ^{-l-1} n\right) \\
& \quad=P\left(\left|\int_{0}^{1} g_{\epsilon}[G(s)-G(t)] d s-L_{G}(1, G(t))\right| \geq \log ^{-l-1} n\right)=o(1),
\end{aligned}
$$

as $n \rightarrow \infty$. This, together with Assumption 3.2 and the fact that $\left|g_{\epsilon}(y)-g_{\epsilon}(z)\right| \leq \epsilon^{-2}$ $|y-z|$, implies that

$$
\begin{aligned}
\left|\frac{1}{n} \sum_{j=1}^{n} g_{\epsilon}\left(x_{j, n}-x_{[n t], n}\right)-L_{n t}\right| \\
\leq\left|\int_{0}^{1} g_{\epsilon}\left(x_{[n s], n}-x_{[n t], n}\right) d s-\int_{0}^{1} g_{\epsilon}\left[G_{n}(s)-G_{n}(t)\right] d s\right|+2 /(\epsilon n) \\
\quad+\left|\int_{0}^{1} g_{\epsilon}\left[G_{n}(s)-G_{n}(t)\right] d s-L_{n t}\right| \\
=O_{a . s}\left(\epsilon^{-2} n^{-\delta_{0}}\right)+2 /(\epsilon n)+O_{P}\left(\log ^{-l-1} n\right) \\
\leq O_{P}\left(n^{2 \alpha-\delta_{0}}+\log ^{-l-1} n\right)=o_{P}\left(\log ^{-l} n\right),
\end{aligned}
$$

uniformly for $t \in[0,1]$, as $\alpha<\delta_{0} / 2$. This yields (A.23).

We finally prove (A.22), let $\bar{g}_{\epsilon n}(z)$ be the step function which takes the value $g_{\epsilon}\left(m n^{\zeta} / c_{n}\right)$ for $z \in\left[m n^{\zeta} / c_{n},(m+1) n^{\zeta} / c_{n}\right), m \in \mathbb{Z}$. It suffices to show that, uniformly for all $1 \leq k \leq n$, (letting $\left.\bar{g}_{j}(y)=\bar{g}\left(c_{n}\left(x_{j, n}-x_{k, n}\right)-y\right)\right)$ :

$$
\begin{aligned}
\Delta_{1 n}(k) & :=\left|\frac{1}{n} \sum_{j=1}^{n} g_{\epsilon}\left(x_{j, n}-x_{k, n}\right)-\frac{1}{n} \sum_{j=1}^{n} \bar{g}_{\epsilon n}\left(x_{j, n}-x_{k, n}\right) \int_{-\infty}^{\infty} \bar{g}_{j}(y) d y\right| \\
& =o_{a . s .}\left(\log ^{-l} n\right), \\
\Delta_{2 n}(k) & :=\left|\frac{1}{n} \sum_{j=1}^{n} \bar{g}_{\epsilon n}\left(x_{j, n}-x_{k, n}\right) \int_{-\infty}^{\infty} \bar{g}_{j}(y) d y-\int_{-\infty}^{\infty} \frac{1}{n} \sum_{j=1}^{n} g_{\epsilon}\left(y / c_{n}\right) \bar{g}_{j}(y) d y\right| \\
& =o_{a . s .}\left(\log ^{-l} n\right), \\
\Delta_{3 n}(k) & :=\left|\int_{-\infty}^{\infty} \frac{1}{n} \sum_{j=1}^{n} g_{\epsilon}\left(y / c_{n}\right) \bar{g}_{j}(y) d y-\frac{c_{n}}{n} \sum_{j=1}^{n} \bar{g}\left[c_{n}\left(x_{j, n}-x_{k, n}\right)\right]\right| \\
& =o_{a . s .}(\log -l n) .
\end{aligned}
$$

In fact, by noting that $\left|g_{\epsilon}(y)-g_{\epsilon}(z)\right| \leq \epsilon^{-2}|y-z|$ and

$$
\begin{aligned}
\left|\bar{g}_{\epsilon n}(y)-g_{\epsilon}(z)\right| & \leq\left|\bar{g}_{\epsilon n}(y)-g_{\epsilon}(y)\right|+\left|g_{\epsilon}(y)-g_{\epsilon}(z)\right| \\
& \leq C \epsilon^{-2}\left(n^{\zeta} / c_{n}+|y-z|\right),
\end{aligned}
$$

(A.25) follows from that, uniformly for all $1 \leq j, k \leq n$, 


$$
\begin{aligned}
& \left|g_{\epsilon}\left(x_{j, n}-x_{k, n}\right)-\bar{g}_{\epsilon n}\left(x_{j, n}-x_{k, n}\right) \int_{-\infty}^{\infty} \bar{g}_{j}(y) d y\right| \\
& \quad \leq\left|g_{\epsilon}\left(x_{j, n}-x_{k, n}\right)-\bar{g}_{\epsilon n}\left(x_{j, n}-x_{k, n}\right)\right|+\left|\bar{g}_{\epsilon n}\left(x_{j, n}-x_{k, n}\right)\right|\left|1-\int_{-\infty}^{\infty} \bar{g}_{j}(y) d y\right| \\
& \quad \leq C \epsilon^{-2} n^{\zeta} / c_{n}+C_{1} \epsilon^{-1} n^{-\zeta(\gamma-1)}=o_{\text {a.s. }}\left(\log ^{-l} n\right),
\end{aligned}
$$

where we have used the fact that (recalling $\int g(y) d y=1$ ),

$$
\left|1-\int_{-\infty}^{\infty} \bar{g}_{j}(y) d y\right| \leq\left|\int_{-\infty}^{\infty} g(y) I\left\{|y|>n^{\zeta} / 2\right\} d y\right| \leq C n^{-\zeta(\gamma-1)}
$$

due to $\sup _{y}|y|^{\gamma}|g(y)|<\infty$ and $\gamma>1$.

By the definition of $\bar{g}_{j}(y)$ and (A.28) again, (A.26) follows from that, uniformly for all $1 \leq j, k \leq n$,

$$
\begin{aligned}
& \int_{-\infty}^{\infty}\left|\bar{g}_{\epsilon n}\left(x_{j, n}-x_{k, n}\right) \bar{g}_{j}(y)-g_{\epsilon}\left(y / c_{n}\right) \bar{g}_{j}(y)\right| d y \\
& \quad \leq\left(\int_{-\infty}^{\infty} g(y) d y\right)\left(\sup _{y}\left|\bar{g}_{\epsilon n}\left(x_{j, n}-x_{k, n}\right)-g_{\epsilon}\left(y / c_{n}\right)\right| I\left\{\left|c_{n}\left(x_{j, n}-x_{k, n}\right)-y\right| \leq n^{\zeta} / 2\right\}\right) \\
& \quad \leq C \sup _{y}\left[\epsilon^{-2}\left(n^{\zeta} / c_{n}+\left|x_{j, n}-x_{k, n}-y / c_{n}\right|\right) I\left\{\left|x_{j, n}-x_{k, n}-y / c_{n}\right| \leq n^{\zeta} /\left(2 c_{n}\right)\right\}\right] \\
& \quad \leq C \epsilon^{-2}\left(n^{\zeta} / c_{n}\right)=o_{a . s .}\left(\log ^{-l} n\right) .
\end{aligned}
$$

As for (A.27), the result follows from that, by using Lemma A.5,

$$
\begin{aligned}
\Delta_{3 n}(k)= & \left|\int_{-\infty}^{\infty} \frac{1}{n} \sum_{j=1}^{n}\left\{\bar{g}\left[c_{n}\left(x_{j, n}-x_{k, n}\right)-y\right]-\bar{g}\left[c_{n}\left(x_{j, n}-x_{k, n}\right)\right]\right\} g_{\epsilon}\left(y / c_{n}\right) d y\right| \\
\leq & \sup _{t} \sup _{|s-t| \leq c_{n} \epsilon}\left|\frac{c_{n}}{n} \sum_{j=1}^{n}\left\{\bar{g}\left(c_{n} x_{j, n}+s\right)-\bar{g}\left(c_{n} x_{j, n}+t\right)\right\}\right| \\
& \times\left(\frac{1}{c_{n}} \int_{-\infty}^{\infty} g_{\epsilon}\left(y / c_{n}\right) d y\right) \\
= & o_{a . s .}\left(\log ^{-l} n\right)
\end{aligned}
$$

uniformly for $1 \leq k \leq n$.

The proof of Theorem 3.1 is complete.

\section{A.4. Proof of Theorem 3.2}

To prove (3.4), we make use of Theorem 3.1. First note that $K(x)$ satisfies Assumption 3.1 as it has a compact support. Let $x_{k, n}=\frac{x_{k}}{\sqrt{n} \phi}, 1 \leq k \leq n$, where $x_{k}$ satisfies Assumption 2.1 with $\sum_{i=0}^{\infty} i\left|\phi_{i}\right|<\infty$. As shown in Chan and Wang (2014), $x_{k, n}$ satisfies Assumption 3.3. $x_{k, n}$ also satisfies Assumption 3.2. Explicitly we will show later that $\left\{v_{j}, j \in Z\right\}$ can be redefined on a richer probability space which also contains a standard Brownian motion $W_{1}(t)$ and a sequence of stochastic processes $G_{1 n}(t)$ such that $\left\{G_{1 n}(t), 0 \leq t \leq 1\right\}={ }_{D}$ $\left\{G_{1}(t), 0 \leq t \leq 1\right\}$ for each $n \geq 1$ and

$$
\sup _{0 \leq t \leq 1}\left|x_{[n t], n}-G_{1 n}(t)\right|=o\left(n^{-\delta_{0}}\right), \quad \text { a.s., }
$$


for some $\delta_{0}>0$, where $G_{1}(t)=W_{1}(t)+\kappa \int_{0}^{t} e^{\kappa(t-s)} W_{1}(s) d s$. We remark that $G_{1}(t)$ is a continuous semimartingale having a local time $L_{G_{1}}(t, x)$ that satisfies (3.2). To see this claim, it suffices to use Corollary 1.8 of Revuz and Yor (1994, p. 226) with minor modifications.

Due to these facts, it follows from Theorem 3.1 that

$\sup _{0 \leq t \leq 1}\left|\frac{1}{\sqrt{n} h} \sum_{k=1}^{n} K\left[\left(x_{k}-x_{[n t]}\right) / \sqrt{n} \phi\right]-L_{n t}\right|=o_{P}\left(\log ^{-l} n\right)$,

for any $l>0, h \rightarrow 0$, and $n^{1-\epsilon_{0}} h^{2} \rightarrow \infty$ where $\epsilon_{0}>0$ can be taken arbitrarily small and

$L_{n t}=\lim _{\epsilon \rightarrow 0} \frac{1}{2 \epsilon} \int_{0}^{1} I\left(\left|G_{1 n}(s)-G_{1 n}(t)\right| \leq \epsilon\right) d s$.

Note that, for each $n \geq 1,\left\{L_{n t}, 0 \leq t \leq 1\right\}={ }_{D}\left\{L_{t}, 0 \leq t \leq 1\right\}$ due to $\left\{G_{1 n}(t), 0 \leq\right.$ $t \leq 1\}={ }_{D}\left\{G_{1}(t), 0 \leq t \leq 1\right\}$, where $L_{t}=\lim _{\epsilon \rightarrow 0} \frac{1}{2 \epsilon} \int_{0}^{1} I\left(\left|G_{1}(s)-G_{1}(t)\right| \leq \epsilon\right) d s$. The result (3.4) now follows from (A.31) and the well known fact that $P\left(\inf _{0 \leq t \leq 1} L_{t}=0\right)=0$, due to the continuity of the process $G_{1}(s)$.

To end the proof of (3.4), it remains to show (A.30). In fact, the classical strong approximation theorem implies that, on a richer probability space,

$\sup _{0 \leq t \leq 1}\left|\sum_{j=1}^{[n t]} v_{j}-W_{1}(n t)\right|=o\left[n^{1 /(2+\delta)}\right], \quad$ a.s.

See, e.g., Csörgö and Révész (1981). Taking this result into consideration, the same technique as in the proof of Phillips (1987) (see also Chan and Wei, 1987) yields

$\sup _{0 \leq t \leq 1}\left|\sum_{j=1}^{[n t]} \lambda^{[n t]-j} v_{j}-G_{1 n}^{*}(t)\right|=o\left[n^{1 /(2+\delta)}\right], \quad$ a.s.

where $G_{1 n}^{*}(t)=W_{1}(n t)+\kappa \int_{0}^{t} e^{\kappa(t-s)} W_{1}(n s) d s$. Let $G_{1 n}(t)=G_{1 n}^{*}(t) / \sqrt{n}$. It is readily seen that $\left\{G_{1 n}(t), 0 \leq t \leq 1\right\}={ }_{D}\left\{G_{1}(t), 0 \leq t \leq 1\right\}$ due to $\left\{W_{1}(n t) / \sqrt{n}, 0 \leq t \leq 1\right\}={ }_{D}$ $\left\{W_{1}(t), 0 \leq t \leq 1\right\}$. Now, by virtue of (A.6), it follows that

$$
\begin{aligned}
\sup _{0 \leq t \leq 1}\left|x_{[n t], n}-G_{1 n}(t)\right| \leq & \sup _{0 \leq t \leq 1}\left|\frac{a_{[n t]} x_{[n t]}^{\prime}}{\sqrt{n} \phi}-G_{1 n}(t)\right|+\frac{\sup _{0 \leq t \leq 1}\left|x_{[n t]}^{\prime \prime}+x_{[n t]}^{\prime \prime \prime}\right|}{\sqrt{n} \phi} \\
\leq & \frac{C}{\sqrt{n}} \sup _{0 \leq t \leq 1}\left|\left(\sum_{i=0}^{[n t]} \phi_{i} \lambda^{-i}-\phi\right) \sum_{j=1}^{[n t]} \lambda^{[n t]-j} v_{j}\right| \\
& \times \frac{1}{\sqrt{n}} \sup _{0 \leq t \leq 1}\left|\sum_{j=1}^{[n t]} \lambda^{[n t]-j} v_{j}-G_{1 n}^{*}(t)\right|+O_{\text {a.s. }}\left(n^{-\delta / 2(2+\delta)}\right) \\
= & o\left(n^{-\delta_{0}}\right), \quad \text { a.s., }
\end{aligned}
$$


for some $\delta_{0}>0$, where we have used the fact: due to $\max _{1 \leq i \leq n}\left|\lambda^{-i}-1\right| \leq C n^{-1 / 2}$ and $\max _{1 \leq k \leq n}\left|\sum_{j=1}^{k} \lambda^{k-j} v_{j}\right|=o(\sqrt{n \log n}), a . s$., it follows from $\sum_{i=0}^{\infty} i\left|\phi_{i}\right|<\infty$ that

$$
\begin{aligned}
& \sup _{0 \leq t \leq 1}\left|\left(\sum_{i=0}^{[n t]} \phi_{i} \lambda^{-i}-\phi\right) \sum_{j=1}^{[n t]} \lambda^{[n t]-j} v_{j}\right| \\
& \quad \leq C \max _{1 \leq k \leq \sqrt{n}}\left|\sum_{j=1}^{k} \lambda^{k-j} v_{j}\right|+O(\sqrt{n \log n}) \max _{\sqrt{n} \leq k \leq n}\left|\sum_{i=0}^{k} \phi_{i} \lambda^{-i}-\phi\right| \\
& \quad \leq O_{P}\left(n^{1 / 4} \sqrt{\log n}\right)+O(\sqrt{n \log n})\left(\left|\sum_{i=0}^{\sqrt{n}} \phi_{i}\left(\lambda^{-i}-1\right)\right|+\sum_{i=\sqrt{n}}^{\infty}\left|\phi_{i}\right|\right) \\
& \quad=O\left(n^{1 / 4} \sqrt{\log n}\right), \quad \text { a.s. }
\end{aligned}
$$

This proves (A.30) and also completes the proof of (3.4).

We finally prove (3.5). Simple calculations show that

$V_{n} \leq \frac{2}{n} \sum_{t=1}^{n}\left(J_{1 t}^{2}+J_{2 t}^{2}\right)$

where

$J_{1 t}=\frac{\sum_{s=1}^{n}\left[m\left(x_{s}\right)-m\left(x_{t}\right)\right] K\left[\left(x_{s}-x_{t}\right) / h\right]}{\sum_{s=1}^{n} K\left[\left(x_{s}-x_{t}\right) / h\right]}, J_{2 t}=\frac{\sum_{s=1}^{n} u_{s} K\left[\left(x_{s}-x_{t}\right) / h\right]}{\sum_{s=1}^{n} K\left[\left(x_{s}-x_{t}\right) / h\right]}$.

Assumption 2.4(a) implies that, when $\left|x_{s}-x_{t}\right| \leq M h$ and $h$ is sufficiently small,

$\left|m\left(x_{s}\right)-m\left(x_{t}\right)\right| \leq C\left|x_{s}-x_{t}\right|^{\beta}\left(1+\left|x_{t}\right|^{\alpha}\right) \leq C_{1} h^{\beta}\left(1+\left|x_{t}\right|^{\alpha}\right)$,

uniformly on $s, t$. Using this fact and $K$ has a compact support, it follows that

$\frac{1}{n} \sum_{t=1}^{n} J_{1 t}^{2} \leq \frac{C h^{2 \beta}}{n} \sum_{t=1}^{n}\left(1+\left|x_{t}\right|^{2 \alpha}\right)=O_{P}\left(n^{\alpha} h^{2 \beta}\right)$

As for $J_{2 t}$, by recalling $u_{s}=\sum_{k=1}^{s} \rho^{s-k} \epsilon_{k}$, we have

$\frac{1}{n} \sum_{t=1}^{n} J_{2 t}^{2} \leq\left\{\inf _{t=1, \ldots, n} \sum_{s=1}^{n} K\left[\left(x_{s}-x_{t}\right) / h\right]\right\}^{-1} \frac{1}{n} \sum_{t=1}^{n} J_{2 t}^{* 2}$,

where

$$
J_{2 t}^{*}=\frac{\sum_{k=1}^{n} \epsilon_{k} \sum_{s=k}^{n} \rho^{s-k} K\left[\left(x_{s}-x_{t}\right) / h\right]}{\left(\sum_{s=1}^{n} K\left[\left(x_{s}-x_{t}\right) / h\right]\right)^{1 / 2}} .
$$


It follows from Assumption 2.2 that, for $1 \leq t \leq n$,

$$
\begin{aligned}
E\left(J_{2 t}^{* 2} \mid x_{1}, \ldots, x_{n}\right) & \leq \frac{\sum_{k=1}^{n} E\left(\epsilon_{k}^{2} \mid x_{1}, \ldots, x_{n}\right)\left\{\sum_{s=k}^{n} \rho^{s-k} K\left[\left(x_{s}-x_{t}\right) / h\right]\right\}^{2}}{\sum_{s=1}^{n} K\left[\left(x_{s}-x_{t}\right) / h\right]} \\
& \leq \frac{C \sum_{k=1}^{n} \sum_{s=k}^{n} \rho^{s-k} K^{2}\left[\left(x_{s}-x_{t}\right) / h\right] \sum_{s=k}^{n} \rho^{s-k}}{\sum_{s=1}^{n} K\left[\left(x_{s}-x_{t}\right) / h\right]} \\
& \leq C_{1},
\end{aligned}
$$

where we have used the fact that $\sum_{k=0}^{\infty} \rho^{k}=1 /(1-\rho)<\infty$. Hence $\frac{1}{n} \sum_{t=1}^{n} J_{2 t}^{* 2}=$ $O_{P}(1)$. Due to this fact and (3.4), we get

$\frac{1}{n} \sum_{t=1}^{n} J_{2 t}^{2}=O_{P}\left[\left(n h^{2}\right)^{-1 / 2}\right]$.

Combining (A.34)-(A.36), we prove (3.5) and hence complete the proof of Theorem 3.2. 